\title{
A Generalized Approach towards Soft Expert Sets via Neutrosophic Cubic Sets with Applications in Games
}

\author{
Muhammad Gulistan ${ }^{1}\left[\right.$ and Nasruddin Hassan ${ }^{2, *}$ \\ 1 Department of Mathematics \& Statistics, Hazara University, Mansehra 21130, Pakistan; \\ gulistanmath@hu.edu.pk or gulistanm21@yahoo.com \\ 2 School of Mathematical Sciences, Faculty of Science and Technology, Universiti Kebangsaan Malaysia, \\ Bangi 43600, Selangor, Malaysia \\ * Correspondence: nas@ukm.edu.my; Tel.: +60-1-9214-5750
}

Received: 28 January 2019; Accepted: 19 February 2019; Published: 22 February 2019

\begin{abstract}
Games are considered to be the most attractive and healthy event between nations and peoples. Soft expert sets are helpful for capturing uncertain and vague information. By contrast, neutrosophic set is a tri-component logic set, thus it can deal with uncertain, indeterminate, and incompatible information where the indeterminacy is quantified explicitly and truth membership, indeterminacy membership, and falsity membership independent of each other. Subsequently, we develop a combined approach and extend this concept further to introduce the notion of the neutrosophic cubic soft expert sets (NCSESs) by using the concept of neutrosophic cubic soft sets, which is a powerful tool for handling uncertain information in many problems and especially in games. Then we define and analyze the properties of internal neutrosophic cubic soft expert sets (INCSESs) and external neutrosophic cubic soft expert sets (ENCSESs), $P$-order, $P$-union, $P$-intersection, $P$-AND, $P$-OR and $R$-order, $R$-union, $R$-intersection, $R$-AND, and $R$-OR of NCSESs. The NCSESs satisfy the laws of commutativity, associativity, De Morgan, distributivity, idempotentency, and absorption. We derive some conditions for $P$-union and $P$-intersection of two INCSESs to be an INCSES. It is shown that $P$-union and $P$-intersection of ENCSESs need not be an ENCSES. The $R$-union and $R$-intersection of the INCSESs (resp., ENCSESs) need not be an INCSES (resp. ENCSES). Necessary conditions for the $P$-union, $R$-union and $R$-intersection of two ENCSESs to be an ENCSES are obtained. We also study the conditions for $R$-intersection and $P$-intersection of two NCSESs to be an INCSES and ENCSES. Finally, for its applications in games, we use the developed procedure to analyze the cricket series between Pakistan and India. It is shown that the proposed method is suitable to be used for decision-making, and as good as or better when compared to existing models.
\end{abstract}

Keywords: neutrosophic sets; cubic sets; soft sets; neutrosophic cubic soft sets; neutrosophic cubic soft expert system; multicriteria decision-making

\section{Introduction}

Researchers always try to discover methods to handle imprecise and vague information, which is not possible using classical set theory. In this regard, Zadeh gave the concept of fuzzy set [1], to cope with uncertainty. However, fuzzy sets were considered imperfect since it is not always easy to give an exact degree of membership to any element. To overcome this problem, the interval-valued fuzzy set was proposed by Turksen [2]. Atanassov [3] extended the notion of fuzzy sets to intuitionistic fuzzy sets by introducing the non-membership of an element with its membership in a set $X$, which were proven to be a better tool than fuzzy sets. Furthermore, the intuitionistic fuzzy sets are used in many directions [4]. Smarandache gave the notion of neutrosophic sets as a generalization of 
intuitionistic fuzzy sets and fuzzy sets [5]. The idea of neutrosophic sets are further expanded to different directions [6-9] by various researchers. Jun et al. [10] gave the idea of cubic set and it was characterized by interval-valued fuzzy set and fuzzy set, which is a more general tool to capture uncertainty and vagueness, since fuzzy set deals with single-value membership while interval-valued fuzzy set ranges the membership in the form of intervals. The hybrid platform provided by cubic set has the main advantage since it contains more information than a fuzzy set and interval-valued fuzzy set. By using this concept, different problems arising in several areas can be solved by choosing the finest choice by means of cubic sets as in the works of Abughazalah and Yaqoob [11], Rashid et al. [12], Gulistan et al. [13], Ma et al. [14], Naveed at al. [15], Gulistan et al. [16], Khan et al. [17,18], Yaqoob et al. [19], and Aslam et al. [20].

More recently, Jun et al. [21] gave the idea of neutrosophic cubic set and it was subsequently used in many areas by Khan et al. [22] and Gulistan et al. [23,24].

On the other hand, Molodtsov [25] introduced the concept of soft sets that can be seen as a new mathematical theory for dealing with uncertainty. It was applied to many different fields by Maji et al. [26] who later defined fuzzy soft set theory and some properties of fuzzy soft sets [27]. Hybrids of soft sets were further developed [28-32].

Alkhazaleh and Salleh in 2011 defined the concept of soft expert set in which the user could know the opinion of all the experts in one model and gave an application of this concept in the decision-making problem [33]. Arokia et al. [34] studied fuzzy parameterizations for decision-making in risk management systems via soft expert set. Arokia and Arockiarani [35] provided a fusion of soft expert set and matrix models. Alkhazaleh and Salleh [36] extended the concept of soft expert set in terms of fuzzy set and provided its application. Bashir and Salleh [37] provided the concept of fuzzy parameterized soft expert set. Bashir et al. [38] discussed possibility fuzzy soft expert set. Alhazaymeh et al. [39] provided the application of generalized vague soft expert set in decision-making. Broumi and Smarandache [40] extended the soft expert sets in terms of intuitionistic fuzzy sets. Abu Qamar and Hassan [41,42] presented the idea of Q-neutrosophic soft relation and its entropy measures of distance and similarity. Sahin et al. [43] gave the idea of neutrosophic soft expert sets while Uluçay et al. [44], introduced the concept of generalized neutrosophic soft expert set for multiple-criteria decision-making. Neutrosophic vague soft expert set theory was put forward by Al-Quran and Hassan [45] and developed it further to complex neutrosophic soft expert set [46,47]. Qayyum et al. [48] gave the idea of cubic soft expert sets for a more general approach. Ziemba and Becker [49] presented analysis of the digital divide using fuzzy forecasting, which is a new approach in decision-making.

Hence it is natural to extend the concept of expert sets to neutrosophic cubic soft expert sets for a more generalized approach. The major contribution of this paper is the development of neutrosophic cubic soft expert sets(NCSESs) by using the concept of neutrosophic cubic soft sets which generalizes the concept of fuzzy soft expert sets, intuitionistic soft expert sets, and cubic soft expert sets. We define and analyze the properties of internal neutrosophic cubic soft expert sets (INCSESs) and external neutrosophic cubic soft expert sets (ENCSESs), $P$-order, $P$-union, $P$-intersection, $P$-AND, $P$-OR, and $R$-order, $R$-union, $R$-intersection, $R$-AND, and $R$-OR of NCSESs. The NCSESs satisfy the laws of commutativity, associativity, De Morgan, distributivity, idempotentency, and absorption. We derive some conditions for $P$-union and $P$-intersection of two INCSESs to be an INCSES. It is shown that $P$-union and $P$-intersection of ENCSESs need not be an ENCSES. The $R$-union and $R$-intersection of the INCSESs (resp., ENCSESs) need not be an INCSES (resp. ENCSES). Necessary conditions for the $P$-union, $R$-union, and $R$-intersection of two ENCSESs to be an ENCSES are obtained. We also study the conditions for $R$-intersection and $P$-intersection of two NCSESs to be an INCSES and ENCSES. This paper is organized as follows. Section 2 will be on preliminaries, while Section 3 develops an approach to neutrosophic cubic soft expert set. We focus on the basic operations, namely $P$-order, $R$-order, $P$-containment, $R$-containment, $P$-union, $P$-intersection, $R$-union, $R$-intersection, complement, $P$-AND, $P$-OR, $R$-AND, and $R$-OR of NCSESs. Section 4 will present more results on NCSESs, 
followed by Section 5 on application in analyzing a cricket series. A comparison analysis will be discussed in Section 6 and a conclusion is drawn in Section 7.

\section{Preliminaries}

Here we recall some of the basic material from the literature to develop the new theory. For simplicity, the symbol $\left(F_{S}^{E}, A\right)$ stands for the soft expert set, $N$ stands for the neutrosophic set, $I^{N}$ stands for the interval neutrosophic set and $(N C)$ for the neutrosophic cubic sets.

In psychology, decision-making (also spelled decision-making) is regarded as the cognitive process resulting in the selection of a belief or a course of action among several alternative possibilities. Every decision-making process produces a final choice, which may or may not prompt action. Decision-making is the process of identifying and choosing alternatives based on the values, preferences, and beliefs of the decision-maker. Experts set is a technique used in decision-making problems, which is further extended to generalized forms, such as fuzzy experts set, intuitionistic fuzzy expert set, cubic expert sets, neutrosophic expert set and other hybrids. We begin by stating the definition of expert set.

Definition 1. [33] Let $U$ be a universe, $E$ be a set of parameters, and $X$ be a set of experts. Let $O=\{0=$ disagree, 1 =agree $\}$ be a set of two valued opinion, $Z=E \times X \times O$ and $A \subseteq Z$. A pair $\left(F_{S}^{E}, A\right)$ is called a soft expert set over $U$, where $F_{S}^{E}$ is a mapping given by $F_{S}^{E}: A \longrightarrow P(U)$ where $P(U)$ denotes the power set of $U$.

Definition 2. [33] Two soft expert sets $\left(F_{S}^{E}, A\right)$ and $\left(G_{S}^{E}, B\right)$ over $U,\left(F_{S}^{E}, A\right) \subseteq\left(G_{S}^{E}, B\right)$ if

$$
H_{S}^{E}(a)=\left\{\begin{array}{c}
A \subseteq B \\
F_{S}^{E}(a) \subseteq G_{S}^{E}(a) \text { for all } a \in A
\end{array}\right.
$$

and $\left(F_{S}^{E}, A\right)=\left(G_{S}^{E}, B\right)$ if and only if $\left(F_{S}^{E}, A\right) \subseteq\left(G_{S}^{E}, B\right)$ as well as $\left(G_{S}^{E}, B\right) \subseteq\left(F_{S}^{E}, A\right)$.

Definition 3. [33] Let $E$ be a set of parameters and $X$ be a set of experts. The NOT set II $Z$ of $Z=E \times X \times O$ is defined by

$$
\hat{I} Z=\left\{\left(\hat{I} e_{i}, x_{i}, o_{k}\right) . \dot{I} e_{i} \notin E, x_{j} \in X \text { and } o_{k} \in O \forall i, j, k\right\}
$$

Definition 4. [33] The complement of a soft expert set $\left(F_{S}^{E}, A\right)$ is denoted by $\left(F_{S}^{E}, A\right)^{c}=\left(F_{S}^{E c}, I A\right)$ where $F_{S}^{E c}: I A \longrightarrow P(U)$ is a mapping given by $F_{S}^{E c}(a)=U-F_{S}^{E}(I)$ I $)$, for all $a \in I A$.

Definition 5. [33] If $Z=E \times X \times\{1\}$ in Definition 1 then $\left(F_{S}^{E}, A\right)$ is called agree soft expert set over $U$ and it is denoted by $\left(F_{S}^{E}, A\right)_{1}$.

Definition 6. [33] If $Z=E \times X \times\{0\}$ in Definition 1 then $\left(F_{S}^{E}, A\right)$ is called disagree soft expert set over $U$ and it is denoted by $\left(F_{S}^{E}, A\right)_{0}$

Definition 7. [33] The union of two soft expert sets $\left(F_{S}^{E}, A\right)$ and $\left(G_{S}^{E}, B\right)$ over $U$ denoted by $\left(F_{S}^{E}, A\right) \cup$ $\left(G_{S}^{E}, B\right)$, is the soft expert set $\left(H_{S}^{E}, C\right)$ where $C=A \cup B$, and for all $a \in C$,

$$
H_{S}^{E}(a)=\left\{\begin{array}{c}
F_{S}^{E}(a) \text { if } a \in A-B \\
G_{S}^{E}(a) \text { if } a \in B-A \\
F_{S}^{E}(a) \cup G_{S}^{E}(a) \text { if } a \in A \cap B .
\end{array}\right.
$$

Definition 8. [33] The intersection of two soft expert sets $\left(F_{S}^{E}, A\right)$ and $\left(G_{S}^{E}, B\right)$ over $U$ denoted by $\left(F_{S}^{E}, A\right) \cap$ $\left(G_{S}^{E}, B\right)$, is the soft expert set $\left(H_{S}^{E}, C\right)$ where $C=A \cap B$, and for all $a \in C$, 


$$
H_{S}^{E}(a)=\left\{\begin{array}{c}
F_{S}^{E}(a) \text { if } a \in A-B \\
G_{S}^{E}(a) \text { if } a \in B-A \\
F_{S}^{E}(a) \cap G_{S}^{E}(a) \text { if } a \in A \cap B .
\end{array}\right.
$$

Definition 9. [33] If $\left(F_{S}^{E}, A\right)$ and $\left(G_{S}^{E}, B\right)$ are two soft expert sets over $U$ then $\left(F_{S}^{E}, A\right) A N D\left(G_{S}^{E}, B\right)$ denoted by $\left(F_{S}^{E}, A\right) \wedge\left(G_{S}^{E}, B\right)$, is defined by

$$
\left(F_{S}^{E}, A\right) \wedge\left(G_{S}^{E}, B\right)=\left(H_{S}^{E}, A \times B\right)
$$

where $H_{S}^{E}(a, b)=F_{S}^{E}(a) \cap G_{S}^{E}(b)$, for all $(a, b) \in A \times B$.

Definition 10. [33] If $\left(F_{S}^{E}, A\right)$ and $\left(G_{S}^{E}, B\right)$ are two soft expert sets then $\left(F_{S}^{E}, A\right) \operatorname{OR}\left(G_{S}^{E}, B\right)$ denoted by $\left(F_{S}^{E}, A\right) \vee\left(G_{S}^{E}, B\right)$ is defined by

$$
\left(F_{S}^{E}, A\right) \vee\left(G_{S}^{E}, B\right)=\left(H_{S}^{E}, A \times B\right)
$$

where $H_{S}^{E}(a, b)=F_{S}^{E}(a) \cup G_{S}^{E}(b)$, for all $(a, b) \in A \times B$.

Definition 11. [5] A neutrosophic set in $X$ is the structure of the form

$$
N:=\left\{\left\langle x, T_{N}(x), I_{N}(x), F_{N}(x)\right\rangle: x \in X\right\}
$$

where $T_{N}, I_{N}, F_{N}: X \rightarrow[0,1]$ such that $0 \leq T_{N}(x)+I_{N}(x)+F_{N}(x) \leq 3$.

Definition 12. [8] An interval neutrosophic set in X is the structure of the form

$$
I^{N}:=\left\{\left\langle x, T_{I^{N}}(x), I_{I^{N}}(x), F_{I^{N}}(x)\right\rangle: x \in X\right\}
$$

where $T_{N}, I_{N}, F_{N}: X \rightarrow D[0,1]$ such that $[0,0] \preceq T_{N}(x)+I_{N}(x)+F_{N}(x) \preceq[3,3]$.

Definition 13. [21] A neutrosophic cubic set in $X$ is a pair $(N C)=\left(I^{N}, N\right)$ where

$$
I^{N}:=\left\{\left\langle x, T_{I^{N}}(x), I_{I^{N}}(x), F_{I^{N}}(x)\right\rangle: x \in X\right\}
$$

is an interval neutrosophic set in $X$ where $T_{I^{N}}, I_{I^{N}}, F_{I^{N}}: X \rightarrow D[0,1]$ and

$$
N:=\left\{\left\langle x, T_{N}(x), I_{N}(x), F_{N}(x)\right\rangle: x \in X\right\}
$$

is a neutrosophic set in $X$ where $T_{N}, I_{N}, F_{N}: X \rightarrow[0,1]$.

\section{Neutrosophic Cubic Soft Expert Set}

In this section, we develop an approach to neutrosophic cubic soft expert set which is a more general approach for soft expert set theory. We focus on the basic operations namely, $P$-order, $R$-order, $P$-containment, $R$-containment, $P$-union, $P$-intersection, $R$-union, $R$-intersection, complement, $P$-AND, $P$-OR, $R$-AND, and $R$-OR of neutrosophic cubic soft expert sets. The symbol $\left((N C)_{S}^{E}, E, X\right)$ stands for the neutrosophic cubic soft expert set.

Definition 14. Let $U$ be a finite set containing $n$ alternatives, $E$ be a set of criteria, $X$ be a set of experts. A triplet $\left((N C)_{S}^{E}, E, X\right)$ is called neutrosophic cubic soft expert set over $U$, if and only if $(N C)_{S}^{E}: E \times X \rightarrow N C P(U)$ is a mapping into the set of all neutrosophic cubic set in $U$ and defined as

$$
\left((N C)_{S}^{E}, E, X\right)=\left\{(N C)_{S}^{E}(e, x)=\left\{\left\langle u, I_{(e, x)}^{N}(u), N_{(e, x)}(u)\right\rangle, u \in U\right\},(e, x) \in E \times X\right\},
$$


where

$$
I_{(e, x)}^{N}(u)=\left\{\left(u, \widetilde{T}_{I^{N}}(u), \widetilde{I}_{I^{N}}(u), \widetilde{F}_{I^{N}}(u)\right)\right\}, N_{(e, x)}(x)=\left\{\left(u, T_{N}(u), I_{N}(u), F_{N}(u)\right\},\right.
$$

such that

$$
[0,0] \preceq \widetilde{T}_{I^{N}}(u)+\widetilde{I}_{I^{N}}(u)+\widetilde{F}_{I^{N}}(u) \preceq[3,3], \quad 0 \leq T_{N}(u)+I_{N}(u)+F_{N}(u) \leq 3 .
$$

Example 1. Let $U=\left\{u_{1}=\right.$ India, $u_{2}=$ Pakistan $\}$ be the set of countries playing a cricket series, $E=\left\{e_{1}=\right.$ playing conditions, $e_{2}=$ historic record $\}$ be the set of factors affecting the series, $X=\left\{x_{1}, x_{2}, x_{3}\right\}$ be the set of experts giving their expert opinion. Let $E \times X=\left\{\left(e_{1}, x_{1}\right),\left(e_{1}, x_{2}\right),\left(e_{2}, x_{1}\right),\left(e_{2}, x_{2}\right)\right\}$. Then the neutrosophic cubic soft expert set $\left((N C)_{S}^{E}, E, X\right)$ is given by

$$
\begin{aligned}
& (N C)_{S}^{E}\left(e_{1}, x_{1}\right)=\left\{\begin{array}{l}
\left(u_{1},[0.5,0.6],[0.2,0.3],[0.1,0.2], 0.1,0.4,0.5\right), \\
\left(u_{2},[0.6,0.9],[0.6,0.9],[0.6,0.9], 0.9,0.7,0.6\right),
\end{array}\right\} \\
& (N C)_{S}^{E}\left(e_{1}, x_{2}\right)=\left\{\begin{array}{l}
\left(u_{1},[0.5,0.6],[0.2,0.3],[0.1,0.2], 0.1,0.4,0.5\right), \\
\left(u_{2},[0.6,0.9],[0.6,0.9],[0.6,0.9], 0.9,0.7,0.6\right),
\end{array}\right\} \\
& (N C)_{S}^{E}\left(e_{1}, x_{3}\right)=\left\{\begin{array}{l}
\left(u_{1},[0.5,0.6],[0.2,0.3],[0.1,0.2], 0.4,0.3,0.5\right), \\
\left(u_{2},[0.6,0.9],[0.6,0.9],[0.6,0.9], 0.9,0.7,0.6\right),
\end{array}\right\} \\
& (N C)_{S}^{E}\left(e_{2}, x_{1}\right)=\left\{\begin{array}{l}
\left(u_{1},[0.6,0.9],[0.6,0.9],[0.6,0.9], 0.9,0.7,0.6\right), \\
\left(u_{2},[0.6,0.9],[0.6,0.9],[0.6,0.9], 0.9,0.7,0.6\right),
\end{array}\right\} \\
& (N C)_{S}^{E}\left(e_{2}, x_{2}\right)=\left\{\begin{array}{l}
\left(u_{1},[0.6,0.9],[0.6,0.9],[0.6,0.9], 0.9,0.7,0.6\right), \\
\left(u_{2},[0.6,0.9],[0.6,0.9],[0.6,0.9], 0.9,0.7,0.6\right),
\end{array}\right\} \\
& (N C)_{S}^{E}\left(e_{2}, x_{3}\right)=\left\{\begin{array}{l}
\left(u_{1},[0.6,0.9],[0.6,0.9],[0.6,0.9], 0.9,0.7,0.6\right), \\
\left(u_{2},[0.6,0.9],[0.6,0.9],[0.6,0.9], 0.9,0.7,0.6\right),
\end{array}\right\}
\end{aligned}
$$

The function of the form $\left(\widetilde{T}_{I^{N}}(u), T_{N}(u)\right)$ denotes the range of values where the experts are sure to give certain membership to a certain element, $\left(\widetilde{I}_{I^{N}}(u), I_{N}(u)\right)$ denotes the range of values where the experts are hesitant and $\left(\widetilde{F}_{I^{N}}(u), F_{N}(u)\right)$ denotes the range of values where the experts are sure to give negative points to a certain element as a non-membership. Thus, experts have a wide range of scale to make their conclusion as compared to the previous defined versions of fuzzy sets. More specific in the current example is the function of the form $\left(\widetilde{T}_{I^{N}}(u), T_{N}(u)\right)$ which gives the expert opinion for the past performance of these two countries, $\left(\widetilde{I}_{I^{N}}(u), I_{N}(u)\right)$ gives the expert opinion for running series between these two countries and $\left(\widetilde{F}_{I^{N}}(u), F_{N}(u)\right)$ gives the expert opinion for the upcoming series between these two countries which is not to be held in the near future.

Definition 15. A neutrosophic cubic soft expert set

$$
\left((N C)_{S}^{E}, E, X\right)=\left\{(N C)_{S}^{E}(e, x)=\left\{\left\langle u, I_{(e, x)}^{N}(u), N_{(e, x)}(u)\right\rangle, u \in U\right\},(e, x) \in E \times X\right\}
$$

over $U$ is said to be:

(i) Internal truth neutrosophic cubic soft experts set (briefly, ITNCSESs) if for all $u \in U$, so that

$$
T_{I^{N}}^{-}(u) \leq T_{N}(u) \leq T_{I^{N}}^{+}(u), \forall u \in U .
$$

(ii) Internal indeterminacy neutrosophic cubic soft experts set (briefly, IINCSESs) if for all $u \in U$, so that

$$
I_{I^{N}}^{-}(u) \leq I_{N}(u) \leq I_{I^{N}}^{+}(u), \forall u \in U .
$$

(iii) Internal falsity neutrosophic cubic soft experts set (briefly, IFNCSESs) if for all $u \in U$, so that

$$
F_{I^{N}}^{-}(u) \leq F_{N}(u) \leq F_{I^{N}}^{+}(u), \forall u \in U .
$$


If a neutrosophic cubic soft expert set $\left((N C)_{S}^{E}, E, X\right)$ in $X$, satisfies (i), (ii), (iii), then it is known as internal neutrosophic cubic soft expert set in $X$, abbreviated as (INCSESs).

Example 2. Consider the Example 1. Then the internal neutrosophic cubic soft expert set is given by

$$
\begin{aligned}
& (N C)_{S}^{E}\left(e_{1}, x_{1}\right)=\left\{\begin{array}{l}
\left(u_{1},[0.4,0.6],[0.2,0.5],[0.1,0.5], 0.5,0.4,0.3\right), \\
\left(u_{2},[0.6,0.9],[0.5,0.9],[0.6,0.8], 0.7,0.6,0.7\right),
\end{array}\right\} \\
& (N C)_{S}^{E}\left(e_{2}, x_{1}\right)=\left\{\begin{array}{l}
\left(u_{1},[0.5,0.7],[0.2,0.4],[0.1,0.4], 0.6,0.3,0.2\right), \\
\left(u_{2},[0.6,0.9],[0.7,0.9],[0.6,0.8], 0.7,0.8,0.7\right),
\end{array}\right\} \\
& (N C)_{S}^{E}\left(e_{1}, x_{2}\right)=\left\{\begin{array}{l}
\left(u_{1},[0.5,0.8],[0.1,0.3],[0.1,0.4], 0.7,0.2,0.3\right), \\
\left(u_{2},[0.5,0.9],[0.6,0.8],[0.4,0.9], 0.6,0.7,0.6\right),
\end{array}\right\} \\
& (N C)_{S}^{E}\left(e_{2}, x_{2}\right)=\left\{\begin{array}{c}
\left(u_{1},[0.6,0.8],[0.3,0.9],[0.6,0.9], 0.7,0.7,0.8\right), \\
\left(u_{2},[0.3,0.9],[0.7,0.9],[0.6,0.8], 0.6,0.8,0.7\right)
\end{array}\right\}
\end{aligned}
$$

Remark 1. We can draw the following conclusion from Example 2;

(i) If the value of $N_{(e, x)}(u)$ lies in the interval $I_{(e, x)}^{N}(u)$, then it means that the respective team is going to maintain its progress in different time frames.

(ii) If the panel of experts consists of the internal panel (meaning that the experts are from the same country or same cricket board), then it is known as INCSESs.

Definition 16. A neutrosophic cubic soft expert set

$$
\left((N C)_{S}^{E}, E, X\right)=\left\{(N C)_{S}^{E}(e, x)=\left\{\left\langle u, I_{(e, x)}^{N}(u), N_{(e, x)}(u)\right\rangle, u \in U\right\},(e, x) \in E \times X\right\}
$$

over $U$ is said to be:

(i) External truth neutrosophic cubic soft expert set (briefly, ETNCSESs) if for all $u \in U$, we have

$$
T_{N}(u) \notin\left(T_{I^{N}}^{-}(u), T_{I^{N}}^{+}(u)\right), \forall u \in U
$$

(ii) External indeterminacy neutrosophic cubic soft expert set (briefly, EINCSESs) if for all $u \in U$, we have

$$
I_{N}(x) \notin\left(I_{I^{N}}^{-}(x), I_{I^{N}}^{+}(x)\right), \forall u \in U
$$

(iii) External falsity neutrosophic cubic soft expert set (briefly, EFNCSESs) if for all $u \in U$, we have

$$
F_{N}(x) \notin\left(F_{I^{N}}^{-}(x), F_{I^{N}}^{+}(x)\right), \forall u \in U
$$

If a neutrosophic cubic soft expert set $\left((N C)_{S}^{E}, E, X\right)$ over $U$, satisfies (i), (ii), (iii), then it is known as external neutrosophic cubic soft expert set in $X$, abbreviated as (ENCSESs).

Example 3. Let $U$ be the set of countries playing a one-day international (ODI) triangular series provided in Example 1, then the external neutrosophic cubic soft expert set is given by; 


$$
\begin{aligned}
& (N C)_{S}^{E}\left(e_{1}, x_{1}\right)=\left\{\begin{array}{l}
\left(u_{1},[0.4,0.6],[0.2,0.5],[0.1,0.5], 0.3,0.1,0.7\right), \\
\left(u_{2},[0.6,0.7],[0.5,0.6],[0.6,0.8], 0.8,0.7,0.9\right),
\end{array}\right\} \\
& (N C)_{S}^{E}\left(e_{2}, x_{1}\right)=\left\{\begin{array}{l}
\left(u_{1},[0.5,0.7],[0.2,0.4],[0.1,0.4], 0.8,0.5,0.6\right), \\
\left(u_{2},[0.4,0.6],[0.3,0.5],[0.6,0.8], 0.7,0.2,0.4\right),
\end{array}\right\} \\
& (N C)_{S}^{E}\left(e_{1}, x_{2}\right)=\left\{\begin{array}{l}
\left(u_{1},[0.5,0.8],[0.1,0.3],[0.1,0.4], 0.4,0.4,0.5\right), \\
\left(u_{2},[0.1,0.3],[0.6,0.8],[0.4,0.6], 0.6,0.5,0.3\right),
\end{array}\right\} \\
& (N C)_{S}^{E}\left(e_{2}, x_{2}\right)=\left\{\begin{array}{l}
\left(u_{1},[0.6,0.7],[0.3,0.4],[0.6,0.8], 0.8,0.7,0.5\right), \\
\left(u_{2},[0.3,0.5],[0.7,0.8],[0.6,0.7], 0.6,0.5,0.8\right),
\end{array}\right\}
\end{aligned}
$$

Remark 2. We can draw the following conclusion from Example 3;

(i) If the value of $N_{(e, x)}(u)$ does not lie in the interval $I_{(e, x)}^{N}(u)$, then it means the respective team is not maintaining its progress in different time frames.

(ii) If the panel of experts consists of the external panel (meaning that the experts are not from the same country or same cricket board), then it is known as ENCSESs.

Our next discussion is to define some basic operations on neutrosophic cubic soft expert sets to get more insight of neutrosophic cubic soft expert sets.

Definition 17. A NCSESS $\left((N C)_{S 1}^{E}, E_{1}, X_{1}\right)$ over $U$ is said to be P-order contained in another NCSESs $\left((N C)_{S 2}^{E}, E_{2}, X_{2}\right)$ over $U$, denoted by $\left((N C)_{S 1}^{E}, E_{1}, X_{1}\right) \subseteq_{P}\left((N C)_{S 2}^{E}, E_{2}, X_{2}\right)$,

if (i) $E_{1} \subseteq E_{2}$,

(ii) $X_{1} \subseteq X_{2}$,

(iii) $(N C)_{S 1}^{E}(e, x) \subseteq_{P}(N C)_{S 2}^{E}(e, x)$ for all $e \in E_{1}, x \in X_{1}$, where condition (iii) implies that

$$
I_{1(N C))_{S}^{E}\left(e_{1}, x_{1}\right)}^{N}(x) \preceq I_{2(N C)_{S}^{E}\left(e_{2}, x_{2}\right)}^{N}(x), \quad N_{1(N C)_{S}^{E}\left(e_{1}, x_{1}\right)}(x) \leq N_{2(N C)_{S}^{E}\left(e_{2}, x_{2}\right)}(x) .
$$

Definition 18. A NCSESS $\left((N C)_{S 1}^{E}, E_{1}, X_{1}\right)$ over $U$ is said to be R-order contained in another NCSES $\left((N C)_{S 2}^{E}, E_{2}, X_{2}\right)$ over $U$, denoted by $\left((N C)_{S 1}^{E}, E_{1}, X_{1}\right) \subseteq_{R}\left((N C)_{S 2}^{E}, E_{2}, X_{2}\right)$,

if (i) $E_{1} \subseteq E_{2}$,

(ii) $X_{1} \subseteq X_{2}$,

(iii) $(N C)_{S 1}^{E}(e, x) \subseteq_{R}(N C)_{S 2}^{E}(e, x)$ for all $e \in E_{1}, x \in X_{1}$,

where condition (iii) implies that

$$
I_{1(N C))_{S}^{E}\left(e_{1}, x_{1}\right)}^{N}(x) \preceq I_{2(N C))_{S}^{E}\left(e_{2}, x_{2}\right)}^{N}(x), \quad N_{1(N C)_{S}^{E}\left(e_{1}, x_{1}\right)}(x) \geq N_{2(N C))_{S}^{E}\left(e_{2}, x_{2}\right)}(x) .
$$

Definition 19. Two NCSESs $\left((N C)_{S 1}^{E}, E_{1}, X_{1}\right)$ and $\left((N C)_{S 2}^{E}, E_{2}, X_{2}\right)$ over $U$ is said to be equal which is denoted by $\left((N C)_{S 1}^{E}, E_{1}, X_{1}\right)=\left((N C)_{S 2}^{E}, E_{2}, X_{2}\right)$,

if (i) $A=B$,

(ii) $X_{1}=X_{2}$,

(iii) $(N C)_{S 1}^{E}(e)=(N C)_{S 2}^{E}(e)$ for all $e \in E=A=B, x \in X=X_{1}=X_{2}$,

where condition (iii) implies that

$$
I_{1(N C))_{S}^{E}\left(e_{1}, x_{1}\right)}^{N}(x)=I_{2(N C))_{S}^{E}\left(e_{2}, x_{2}\right)}^{N}(x), \quad N_{1(N C))_{S}^{E}\left(e_{1}, x_{1}\right)}(x)=N_{2(N C))_{S}^{E}\left(e_{2}, x_{2}\right)}(x) .
$$

Remark 3. (a) We observe from Definitions 17-19, that for any two NCSESs $\left((N C)_{S 1}^{E}, E_{1}, X_{1}\right)$ and $\left((N C)_{S 2}^{E}, E_{2}, X_{2}\right)$ over $U$;

(i) If $\left((N C)_{S 1}^{E}, E_{1}, X_{1}\right) \subseteq_{P}\left((N C)_{S 2}^{E}, E_{2}, X_{2}\right)$ and $\left((N C)_{S 2}^{E}, E_{2}, X_{2}\right) \subseteq_{P}\left((N C)_{S 1}^{E}, E_{1}, X_{1}\right)$, 
then $\left((N C)_{S 1}^{E}, E_{1}, X_{1}\right)=\left((N C)_{S 2}^{E}, E_{2}, X_{2}\right)$,

(ii) If $\left((N C)_{S 1}^{E}, E_{1}, X_{1}\right) \subseteq_{R}\left((N C)_{S 2}^{E}, E_{2}, X_{2}\right)$ and $\left((N C)_{S 2}^{E}, E_{2}, X_{2}\right) \subseteq_{R}\left((N C)_{S 1}^{E}, E_{1}, X_{1}\right)$,

then $\left((N C)_{S 1}^{E}, E_{1}, X_{1}\right)=\left((N C)_{S 2}^{E}, E_{2}, X_{2}\right)$.

(b) Using Definitions 17-19, one can easily compare the performance of two cricket teams in different time frames.

Definition 20. Let $\left((N C)_{S 1}^{E}, E_{1}, X_{1}\right)$ and $\left((N C)_{S 2}^{E}, E_{2}, X_{2}\right)$ be two NCSESs in $U$.

Then we define (i) $\left((N C)_{S 1}^{E}, E_{1}, X_{1}\right) \cup_{P}\left((N C)_{S 2}^{E}, E_{2}, X_{2}\right)=\left((N C)_{S 3}^{E}, E_{3}, X_{3}\right)$, where $E_{3}=E_{1} \cup$ $E_{2}, X_{3}=X_{1} \cup X_{2}$

$$
(N C)_{S 3}^{E}\left(e_{i}\right)=\left\{\begin{array}{cc}
(N C)_{S 1}^{E}\left(e_{i}\right) & \text { if } e_{i} \in E_{1}-E_{2} \\
(N C)_{S 2}^{E}\left(e_{i}\right) & \text { if } e_{i} \in E_{2}-E_{1} \\
(N C)_{S 1}^{E}\left(e_{i}\right) \vee_{P}(N C)_{S 2}^{E}\left(e_{i}\right) \text { if } e_{i} \in E_{1} \cap E_{2}
\end{array}\right.
$$

where

$$
\begin{aligned}
& (N C)_{S 1}^{E}\left(e_{i}\right) \vee_{P}(N C)_{S 2}^{E}\left(e_{i}\right)=\left\{\left\langle u, I_{(N C)_{S 1}^{E}\left(e_{i}\right)}^{N} \vee I_{(N C)_{S 2}^{E}\left(e_{i}\right)}^{N}, N_{(N C))_{S 1}^{E}\left(e_{i}\right)} \vee N_{(N C))_{S 2}^{E}\left(e_{i}\right)}\right\rangle: u \in U\right\}
\end{aligned}
$$

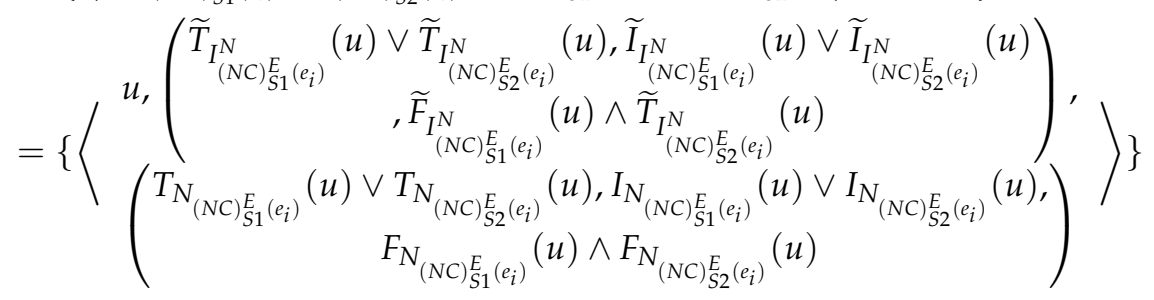

$$
\begin{aligned}
& \text { (ii) }\left((N C)_{S 1}^{E}, E_{1}, X_{1}\right) \cap_{P}\left((N C)_{S 2}^{E}, E_{2}, X_{2}\right)=\left((N C)_{S 3}^{E}, E_{3}, X_{3}\right) \text {, where } E_{3}=E_{1} \cap E_{2}, X_{3}=X_{1} \cap X_{2} \\
& (N C)_{S 3}^{E}\left(e_{i}\right)=\left\{(N C)_{S 1}^{E}\left(e_{i}\right) \wedge_{P}(N C)_{S 2}^{E}\left(e_{i}\right) \text { if } e_{i} \in E_{1} \cap E_{2}\right.
\end{aligned}
$$

where

$$
\begin{aligned}
& (N C)_{S 1}^{E}\left(e_{i}\right) \wedge_{P}(N C)_{S 2}^{E}\left(e_{i}\right)=\left\{\left\langle u, I_{(N C)_{S 1}^{E}\left(e_{i}\right)}^{N} \wedge I_{(N C))_{S 2}^{E}\left(e_{i}\right)}^{N}, N_{(N C))_{S 1}^{E}\left(e_{i}\right)} \wedge N_{(N C))_{S 2}^{E}\left(e_{i}\right)}\right\rangle: u \in U\right\}
\end{aligned}
$$

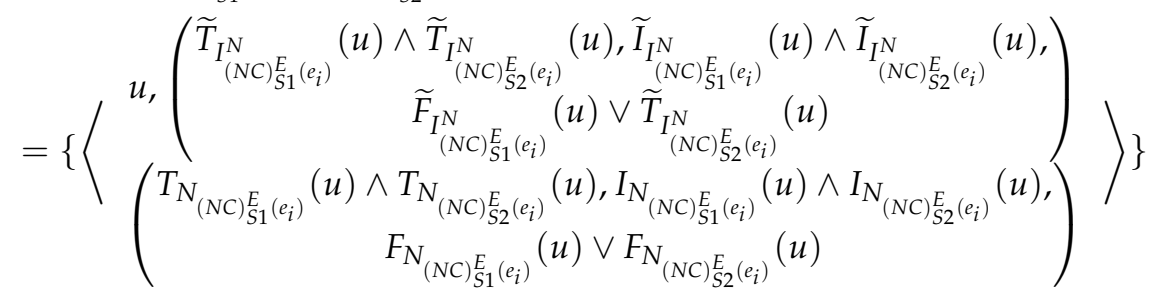

(iii) $\left((N C)_{S 1}^{E}, E_{1}, X_{1}\right) \cup_{R}\left((N C)_{S 2}^{E}, E_{2}, X_{2}\right)=\left((N C)_{S 3}^{E}, E_{3}, X_{3}\right)$, where $E_{3}=E_{1} \cup E_{2}, X_{3}=X_{1} \cup X_{2}$,

$$
(N C)_{S 3}^{E}\left(e_{i}\right)=\left\{\begin{array}{cc}
(N C)_{S 1}^{E}\left(e_{i}\right) & \text { if } e_{i} \in E_{1}-E_{2} \\
(N C)_{S 2}^{E}\left(e_{i}\right) & \text { if } e_{i} \in E_{2}-E_{1} \\
(N C)_{S 1}^{E}\left(e_{i}\right) \vee_{R}(N C)_{S 2}^{E}\left(e_{i}\right) & \text { if } e_{i} \in E_{1} \cap E_{2}
\end{array}\right.
$$


where

$$
\begin{aligned}
& (N C)_{S 1}^{E}\left(e_{i}\right) \vee_{R}(N C)_{S 2}^{E}\left(e_{i}\right)=\left\{\left\langle u, I_{(N C))_{S 1}^{E}\left(e_{i}\right)}^{N} \vee I_{(N C))_{S 2}^{E}\left(e_{i}\right)}^{N}, N_{(N C))_{S 1}^{E}\left(e_{i}\right)} \wedge N_{(N C))_{S 2}^{E}\left(e_{i}\right)}\right\rangle: u \in U\right\}
\end{aligned}
$$

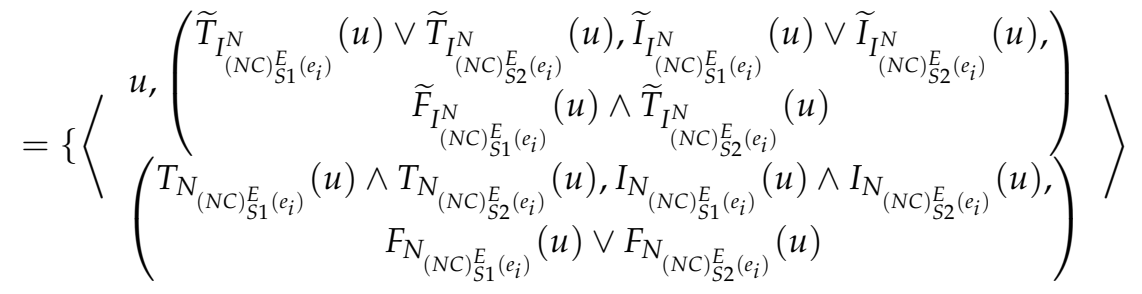

(iv) $\left((N C)_{S 1}^{E}, E_{1}, X_{1}\right) \cap_{R}\left((N C)_{S 2}^{E}, E_{2}, X_{2}\right)=\left((N C)_{S 3}^{E}, E_{3}, X_{3}\right)$, where $E_{3}=E_{1} \cap E_{2}, X_{3}=X_{1} \cap X_{2}$,

$$
(N C)_{S 3}^{E}\left(e_{i}\right)=\left\{(N C)_{S 1}^{E}\left(e_{i}\right) \wedge_{R}(N C)_{S 2}^{E}\left(e_{i}\right) \text { if } e_{i} \in E_{1} \cap E_{2}\right.
$$

where

$$
\begin{aligned}
& (N C)_{S 1}^{E}\left(e_{i}\right) \wedge_{R}(N C)_{S 2}^{E}\left(e_{i}\right)=\left\{\left\langle u, I_{(N C))_{S 1}^{E}\left(e_{i}\right)}^{N} \wedge I_{(N C)_{S 2}^{E}\left(e_{i}\right)}^{N}, N_{(N C))_{S 1}^{E}\left(e_{i}\right)} \vee N_{(N C))_{S 2}^{E}\left(e_{i}\right)}\right\rangle: u \in U\right\}
\end{aligned}
$$

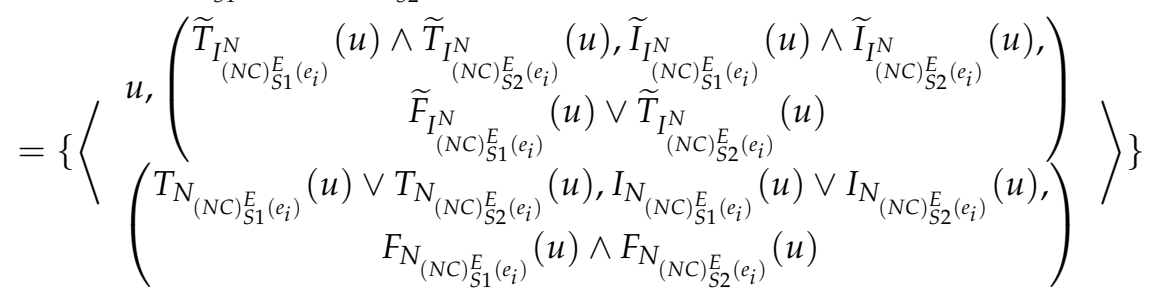

(v) The complement of a neutrosophic cubic soft expert set $\left((N C)_{S}^{E}, E, X\right)$ denoted by

$$
\left((N C)_{S}^{E}, E, X\right)^{c}=\left\{(N C)_{S}^{E c}\left(e_{i}\right)=\left\{\left\langle u, \widetilde{1}-I_{(N C)_{S}^{E}\left(e_{i}\right)}^{N}(u), 1-N_{(N C))_{S}^{E}\left(e_{i}\right)}(u)\right\rangle, u \in U\right\}, e_{i} \in A\right\} .
$$

Proposition 1. Let $\left((N C)_{S 1}^{E}, E_{1}, X_{1}\right),\left((N C)_{S 2}^{E}, E_{2}, X_{2}\right),\left((N C)_{S 3}^{E}, E_{3}, X_{3}\right),\left((N C)_{S 4}^{E}, E_{4}, X_{4}\right)$ be NCSESs in $U$. Then

$$
\begin{aligned}
& \text { (i) If }\left((N C)_{S 1}^{E}, E_{1}, X_{1}\right) \subseteq_{P}\left((N C)_{S 2}^{E}, E_{2}, X_{2}\right) \text { and }\left((N C)_{S 3}^{E}, E_{3}, X_{3}\right) \subseteq_{P}\left((N C)_{S 4}^{E}, E_{4}, X_{4}\right) \\
& \text { then }\left((N C)_{S 1}^{E}, E_{1}, X_{1}\right) \subseteq_{P}\left((N C)_{S 4}^{E}, E_{4}, X_{4}\right) \text {. } \\
& \text { (ii) If }\left((N C)_{S 1}^{E}, E_{1}, X_{1}\right) \subseteq_{P}\left((N C)_{S 2}^{E}, E_{2}, X_{2}\right) \\
& \text { then }\left((N C)_{S 2}^{E}, E_{2}, X_{2}\right)^{C} \subseteq_{P}\left((N C)_{S 1}^{E}, E_{1}, X_{1}\right)^{C} \text {. } \\
& \text { (iii) If }\left((N C)_{S 1}^{E}, E_{1}, X_{1}\right) \subseteq_{P}\left((N C)_{S 2}^{E}, E_{2}, X_{2}\right) \text { and }\left((N C)_{S 1}^{E}, E_{1}, X_{1}\right) \subseteq_{P}\left((N C)_{S 4}^{E}, E_{4}, X_{4}\right) \\
& \text { then }\left((N C)_{S 1}^{E}, E_{1}, X_{1}\right) \subseteq_{P}\left((N C)_{S 2}^{E}, E_{2}, X_{2}\right) \cap_{P}\left((N C)_{S 4}^{E}, E_{4}, X_{4}\right) \text {. } \\
& \text { (iv) If }\left((N C)_{S 1}^{E}, E_{1}, X_{1}\right) \subseteq_{P}\left((N C)_{S 2}^{E}, E_{2}, X_{2}\right) \text { and }\left((N C)_{S 3}^{E}, E_{3}, X_{3}\right) \subseteq_{P}\left((N C)_{S 2}^{E}, E_{2}, X_{2}\right) \\
& \text { then }\left((N C)_{S 1}^{E}, E_{1}, X_{1}\right) \cup_{P}\left((N C)_{S 3}^{E}, E_{3}, X_{3}\right) \subseteq_{P}\left((N C)_{S 2}^{E}, E_{2}, X_{2}\right) \text {. } \\
& \text { (v) If }\left((N C)_{S 1}^{E}, E_{1}, X_{1}\right) \subseteq_{P}\left((N C)_{S 2}^{E}, E_{2}, X_{2}\right) \text { and }\left((N C)_{S 3}^{E}, E_{3}, X_{3}\right) \subseteq_{P}\left((N C)_{S 4}^{E}, E_{4}, X_{4}\right) \\
& \text { then }\left((N C)_{S 1}^{E}, E_{1}, X_{1}\right) \cup_{P}\left((N C)_{S 3}^{E}, E_{3}, X_{3}\right) \subseteq_{P}\left((N C)_{S 2}^{E}, E_{2}, X_{2}\right) \cup_{P}\left((N C)_{S 4}^{E}, E_{4}, X_{4}\right) \text {, } \\
& \text { ((NC) } \left.)_{S 1}^{E}, E_{1}, X_{1}\right) \cap_{P}\left((N C)_{S 3}^{E}, E_{3}, X_{3}\right) \subseteq_{P}\left((N C)_{S 2}^{E}, E_{2}, X_{2}\right) \cap_{P}\left((N C)_{S 4}^{E}, E_{4}, X_{4}\right) \text {. }
\end{aligned}
$$

Proof. The proof is straightforward.

Theorem 1. For any two NCSESS $\left((N C)_{S 1}^{E}, E_{1}, X_{1}\right)$ and $\left((N C)_{S 2}^{E}, E_{2}, X_{2}\right)$ over $U$ the following properties hold:

(i) Idempotent law: $\left((N C)_{S 1}^{E}, E_{1}, X_{1}\right) \cup_{P}\left((N C)_{S 1}^{E}, E_{1}, X_{1}\right)=\left((N C)_{S 1}^{E}, E_{1}, X_{1}\right)$,

$\left((N C)_{S 1}^{E}, E_{1}, X_{1}\right) \cap_{P}\left((N C)_{S 1}^{E}, E_{1}, X_{1}\right)=\left((N C)_{S 1}^{E}, E_{1}, X_{1}\right)$.

(ii) Commutative law: $\left((N C)_{S 1}^{E}, E_{1}, X_{1}\right) \cup_{P}\left((N C)_{S 2}^{E}, E_{2}, X_{2}\right)=\left((N C)_{S 2}^{E}, E_{2}, X_{2}\right) \cup_{P}\left((N C)_{S 1}^{E}, E_{1}, X_{1}\right)$, $\left((N C)_{S 1}^{E}, E_{1}, X_{1}\right) \cap_{P}\left((N C)_{S 2}^{E}, E_{2}, X_{2}\right)=\left((N C)_{S 2}^{E}, E_{2}, X_{2}\right) \cap_{P}\left((N C)_{S 1}^{E}, E_{1}, X_{1}\right)$. 
(iii) Associative law: $\left(\left((N C)_{S 1}^{E}, E_{1}, X_{1}\right) \cup_{P}\left((N C)_{S 2}^{E}, E_{2}, X_{2}\right)\right) \cup_{P}\left((N C)_{S 3}^{E}, E_{3}, X_{3}\right)=\left((N C)_{S 1}^{E}, E_{1}, X_{1}\right)$ $\cup_{P}\left(\left((N C)_{S 2}^{E}, E_{2}, X_{2}\right) \cup_{P}\left((N C)_{S 3}^{E}, E_{3}, X_{3}\right)\right)$,

$\left(\left((N C)_{S 1}^{E}, E_{1}, X_{1}\right) \cap_{P}\left((N C)_{S 2}^{E}, E_{2}, X_{2}\right)\right) \cap_{P}\left((N C)_{S 3}^{E}, E_{3}, X_{3}\right)=\left((N C)_{S 1}^{E}, E_{1}, X_{1}\right) \cap_{P}\left(\left((N C)_{S 2}^{E}, E_{2}, X_{2}\right)\right.$ $\left.\cap_{P}\left((N C)_{S 3}^{E}, E_{3}, X_{3}\right)\right)$.

(iv) Distributive and De Morgan's laws also true.

(v) Involution law: $\left(\left((N C)_{S 1}^{E}, E_{1}, X_{1}\right)^{c}\right)^{c}=\left((N C)_{S 1}^{E}, E_{1}, X_{1}\right)$.

Proposition 2. For any two NCSESs $\left((N C)_{S 1}^{E}, E_{1}, X_{1}\right)$ and $\left((N C)_{S 2}^{E}, E_{2}, X_{2}\right)$ over $U$ the following properties are equivalent:

(i) $\left((N C)_{S 1}^{E}, E_{1}, X_{1}\right) \subseteq_{P}\left((N C)_{S 2}^{E}, E_{2}, X_{2}\right)$,

(ii) $\left((N C)_{S 1}^{E}, E_{1}, X_{1}\right) \cap_{P}\left((N C)_{S 2}^{E}, E_{2}, X_{2}\right)=\left((N C)_{S 1}^{E}, E_{1}, X_{1}\right)$,

(iii) $\left((N C)_{S 1}^{E}, E_{1}, X_{1}\right) \cup_{P}\left((N C)_{S 2}^{E}, E_{2}, X_{2}\right)=\left((N C)_{S 2}^{E}, E_{2}, X_{2}\right)$.

Proof. (i) $\Rightarrow$ (ii) By Definition 20, we have

$$
\left((N C)_{S 1}^{E}, E_{1}, X_{1}\right) \cap_{P}\left((N C)_{S 2}^{E}, E_{2}, X_{2}\right)=\left((N C)_{S 1}^{E} \cap_{P}(N C)_{S 2}^{E}, A \cap B\right)=\left((N C)_{S 1}^{E} \cap_{P}(N C)_{S 2}^{E}, A\right)
$$

as $A \subseteq B$ by hypothesis. Now for any $e \in E_{1}$, since $(N C)_{S 1}^{E}(e) \subseteq_{P}(N C)_{S 2}^{E}(e)$, using Definition 17, implies that $I_{(N C)_{S 1}^{E}\left(e_{i}\right)}^{N}(u) \preceq I_{(N C)_{S 2}^{E}\left(e_{i}\right)}^{N}(u)$ and $N_{(N C)_{S 1}^{E}\left(e_{i}\right)}(u) \leq N_{(N C)_{S 2}^{E}\left(e_{i}\right)}(u)$ for any $u \in U$, where $(N C)_{S 1}^{E}\left(e_{i}\right)=\left\{\left\langle u, I_{(N C))_{S 1}^{E}\left(e_{i}\right)}^{N}(u), N_{(N C))_{S 1}^{E}\left(e_{i}\right)}(u)\right\rangle u \in U\right\}$. Since $I_{(N C))_{S 1}^{N-}\left(e_{i}\right)}^{(u)}(u) I_{(N C))_{S 2}^{E}\left(e_{i}\right)}^{N-}(u)$ and $I_{(N C))_{S 1}^{E}\left(e_{i}\right)}^{N+}(u) \leq I_{(N C))_{S 2}^{N+}\left(e_{i}\right)}^{E}(u)$. Thus

$$
\begin{aligned}
\inf \left\{I_{(N C))_{S 1}^{E}\left(e_{i}\right)}^{N}(u), I_{(N C)_{S 2}^{E}\left(e_{i}\right)}^{N}(u)\right\} & =\left[\inf \left\{I_{(N C))_{S 1}^{E}\left(e_{i}\right)}^{N-}(u) \leq I_{(N C))_{S 2}^{E}\left(e_{i}\right)}^{N-}(u)\right\},\right. \\
\left.\inf \left\{I_{(N C))_{S 1}^{E}\left(e_{i}\right)}^{N+}(u) \leq I_{(N C))_{S 2}^{E}\left(e_{i}\right)}^{N+}(u)\right\}\right] & =\left[I_{(N C))_{S 1}^{N}\left(e_{i}\right)}^{N-}(u) \leq I_{(N C))_{S 1}^{N}\left(e_{i}\right)}^{N+}(u)\right]
\end{aligned}
$$

and $\inf \left\{N_{(N C)_{S 1}^{E}\left(e_{i}\right)}(u), N_{(N C)_{S 2}^{E}\left(e_{i}\right)}(u)\right\}=N_{(N C))_{S 1}^{E}\left(e_{i}\right)}(u)$. So It is ok.

$$
\begin{aligned}
(N C)_{S 1}^{E}(e) \cap_{P}(N C)_{S 2}^{E}(e) & =\left\{\left\langleu, \inf \left\{I_{(N C))_{S 1}^{E}\left(e_{i}\right)}^{N}(u), I_{(N C))_{S 2}^{E}\left(e_{i}\right)}^{N}(u)\right\},\right.\right. \\
\left.\inf \left\{N_{(N C))_{S 1}^{E}\left(e_{i}\right)}(u), N_{(N C))_{S 2}^{E}\left(e_{i}\right)}(u)\right\rangle: u \in U\right\} & =\left\{\left\langle u, I_{(N C))_{S 1}^{E}\left(e_{i}\right)}^{N}(u), N_{(N C))_{S 1}^{E}\left(e_{i}\right)}(u)\right\rangle: u \in U\right\} \\
& =(N C)_{S 1}^{E}(e)
\end{aligned}
$$

Hence $(N C)_{S 1}^{E}(e) \cap_{P}(N C)_{S 2}^{E}(e)=(N C)_{S}^{E}(e)$.

(ii) $\Rightarrow$ (iii) By Definition 20, we have

$$
\left((N C)_{S 1}^{E}, E_{1}, X_{1}\right) \cup_{P}\left((N C)_{S 2}^{E}, E_{2}, X_{2}\right)=\left((N C)_{S 1}^{E} \cup_{P}(N C)_{S 2}^{E}, A \cup B\right)=\left((N C)_{S 1}^{E} \cup_{P}(N C)_{S 2}^{E}, A\right)
$$

as $A \cap A=A$ and $(N C)_{S 2}^{E} \cap(N C)_{S 2}^{E}=(N C)_{S 2}^{E}$, by hypothesis. Now for any $e \in E_{1}$, since $(N C)_{S 1}^{E}(e) \cap_{P}$ $(N C)_{S 2}^{E}(e)=(N C)_{S 1}^{E}(e)$, by Definition 20, we have

$$
\begin{aligned}
\inf \left\{I_{(N C))_{S 1}^{E}\left(e_{i}\right)}^{N}(u), I_{(N C)_{S 2}^{E}\left(e_{i}\right)}^{N}(u)\right\} & =I_{(N C)_{S 1}^{E}\left(e_{i}\right)}^{N}(u) \\
\text { and } \inf \left\{N_{(N C)_{S 1}^{E}\left(e_{i}\right)}(u), N_{(N C)_{S 2}^{E}\left(e_{i}\right)}(u)\right\} & =N_{(N C)_{S 1}^{E}\left(e_{i}\right)}(u)
\end{aligned}
$$

this implies that

$$
\begin{aligned}
\sup \left\{I_{(N C)_{S 1}^{E}\left(e_{i}\right)}^{N}(u), I_{(N C))_{S 2}^{E}\left(e_{i}\right)}^{N}(u)\right\} & =I_{(N C))_{S 2}^{E}\left(e_{i}\right)}^{N}(u) \\
\text { and } \sup \left\{N_{(N C))_{S 1}^{E}\left(e_{i}\right)}(u), N_{(N C))_{S 2}^{E}\left(e_{i 1}\right)}(u)\right\} & =N_{(N C)_{S 2}^{E}\left(e_{i}\right)}(u)
\end{aligned}
$$


Thus, we have

$$
\begin{aligned}
(N C)_{S 1}^{E}(e) \cup_{P}(N C)_{S 2}^{E}(e) & =\left\{\left\langle\begin{array}{c}
u, \sup \left\{I_{(N C))_{S 1}^{E}\left(e_{i}\right)}^{N}(u), I_{(N C))_{S 2}^{E}\left(e_{i}\right)}^{N}(u)\right\}, \\
\sup \left\{N_{(N C))_{S 1}^{E}\left(e_{i}\right)}(u), N_{(N C)_{S 2}\left(e_{i}\right)}(u)\right.
\end{array}\right\rangle: u \in U\right\} \\
& =\left\{\left\langle u, I_{(N C))_{S 2}^{E}\left(e_{i}\right)}^{N}(u), N_{(N C))_{S 2}^{E}\left(e_{i}\right)}(u)\right\rangle: u \in U\right\}=(N C)_{S 2}^{E}(e) .
\end{aligned}
$$

Hence $(N C)_{S 1}^{E}(e) \cap_{P}(N C)_{S 2}^{E}(e)=(N C)_{S 2}^{E}(e)$.

(iii) $\Rightarrow$ (i) By hypothesis we have

$$
\left((N C)_{S 1}^{E}, E_{1}, X_{1}\right) \cup_{P}\left((N C)_{S 2}^{E}, E_{2}, X_{2}\right)=\left((N C)_{S 1}^{E} \cup_{P}(N C)_{S 2}^{E}, A \cup B\right)=\left((N C)_{S 1}^{E} \cup_{P}(N C)_{S 2}^{E}, A\right)
$$

as $A \cup A=A$ and $(N C)_{S 2}^{E} \cup(N C)_{S 2}^{E}=(N C)_{S 2}^{E} \Rightarrow A \subseteq A$ and $(N C)_{S 2}^{E} \subseteq(N C)_{S 2}^{E}$. Also

$$
\begin{aligned}
(N C)_{S 1}^{E}(e) \cup_{P}(N C)_{S 2}^{E}(e) & =\left\{\left\langle\begin{array}{c}
u, \sup \left\{I_{(N C))_{S 1}^{N}\left(e_{i}\right)}^{N}(u), I_{(N C))_{S 2}^{E}\left(e_{i}\right)}^{N}(u)\right\}, \\
\sup \left\{N_{(N C))_{S 1}^{E}\left(e_{i}\right)}(u), N_{(N C))_{S 2}^{E}\left(e_{i}\right)}(u)\right.
\end{array}\right\rangle: u \in U\right\} \\
& =\left\{\left\langle u, I_{(N C))_{S 2}^{E}\left(e_{i}\right)}^{N}(u), N_{(N C))_{S 2}^{E}\left(e_{i}\right)}(u)\right\rangle: u \in U\right\}=(N C)_{S 2}^{E}(e)
\end{aligned}
$$

this implies that $I_{(N C)_{S 1}^{E}\left(e_{i}\right)}^{N}(u) \preceq I_{(N C)_{S 2}^{E}\left(e_{i}\right)}^{N}(u)$ and $N_{(N C)_{S 1}^{E}\left(e_{i}\right)}(u) \leq N_{(N C)_{S 2}^{E}\left(e_{i}\right)}(u)$ for any $u \in U$.

Hence $\left((N C)_{S 1}^{E}, E_{1}, X_{1}\right) \subseteq_{P}\left((N C)_{S 2}^{E}, E_{2}, X_{2}\right)$.

Corollary 1. If we take $X_{1}=X_{2}=X$ in the Proposition 2, then the following are equivalent:

(i) $\left((N C)_{S 1}^{E}, E_{1}, X\right) \subseteq_{P}\left((N C)_{S 2}^{E}, E_{2}, X\right)$,

(ii) $\left((N C)_{S 1}^{E}, E_{1}, X\right) \cap_{P}\left((N C)_{S 2}^{E}, E_{2}, X\right)=\left((N C)_{S 1}^{E}, E_{1}, X\right)$,

(iii) $\left((N C)_{S 1}^{E}, E_{1}, X\right) \cup_{P}\left((N C)_{S 2}^{E}, E_{2}, X\right)=\left((N C)_{S 2}^{E}, E_{2}, X\right)$.

Proof. The proof is straightforward.

\section{More on NCSESs}

In this section, we discuss different types of union and intersection of the NCSESs and their related conditions.

1. The following example shows that R-Union of two INCSESS in $U$ need not be INCSESS in $U$.

Example 4. Let $\left((N C)_{S 1}^{E}, E_{1}, X_{1}\right)$ and $\left((N C)_{S 2}^{E}, E_{2}, X_{2}\right)$ be two INCSESs in $U$, where

$$
\left((N C)_{S 1}^{E}, E_{1}, X_{1}\right)=\left\{I_{(N C))_{S 1}^{E}\left(e_{i}\right)}^{N}=([0.1,0.2],[0.4,0.5],[0.5,0.6]), N_{(N C))_{S 1}^{E}\left(e_{i}\right)}=(0.2,0.3,0.4)\right\}
$$

and

$$
\left((N C)_{S 2}^{E}, E_{2}, X_{2}\right)=\left\{I_{(N C))_{S 2}^{E}\left(e_{i}\right)}^{N}=([0.3,0.4],[0.3,0.5],[0.5,0.7]), N_{(N C))_{S 2}^{E}\left(e_{i}\right)}=(0.4,0.6,0.3)\right\} .
$$

Now by Definition 20, we have $\left((N C)_{S 1}^{E}, E_{1}, X_{1}\right) \cup_{R}\left((N C)_{S 2}^{E}, E_{2}, X_{2}\right)=\left((N C)_{S 3}^{E}, E_{3}, X_{3}\right)$

$$
\left((N C)_{S 3}^{E}, E_{3}, X_{3}\right)=\left\{I_{(N C))_{S 3}^{E}\left(e_{i}\right)}^{N}=\left([0.3,0.4],[0.4,0.5],[0.5,0.7], N_{(N C))_{S 3}^{E}\left(e_{i}\right)}=(0.2,0.3,0.4)\right\}\right.
$$

As $0.2 \notin[0.4,0.5], 0.3 \notin[0.4,0.5]$ and $0.4 \notin[0.5,0.7]$.

Hence $\left((N C)_{S 1}^{E}, E_{1}, X_{1}\right) \cup_{R}\left((N C)_{S 2}^{E}, E_{2}, X_{2}\right)$ is not a INCSES in $U$. 
The following theorem gives the condition under which $R$-union of two INCSESs in $U$ is also a INCSES in $U$.

Theorem 2. Let $\left((N C)_{S 1}^{E}, E_{1}, X_{1}\right)$ and $\left((N C)_{S 2}^{E}, E_{2}, X_{2}\right)$ be two INCSESs in $U$,

such that

$$
\begin{aligned}
& \text { where }\left((N C)_{S 1}^{E}, E_{1}, X_{1}\right)=\left\{(N C)_{S 1}^{E}(e, x)=\left\{\left\langle u, I_{1(e, x)}^{N}(u), N_{1(e, x)}(u)\right\rangle, u \in U\right\},(e, x) \in E_{1} \times X_{1}\right\} \\
& \text { and }\left((N C)_{S 2}^{E}, E_{2}, X_{2}\right)=\left\{(N C)_{S 2}^{E}(f, y)=\left\{\left\langle u, I_{2(f, y)}^{N}(u), N_{2(f, y)}(u)\right\rangle, u \in U\right\},(f, y) \in E_{2} \times X_{2}\right\}
\end{aligned}
$$

$$
\sup \left\{I_{(N C))_{S 1}^{E}\left(e_{i}\right)}^{N-}(u), I_{(N C)_{S 2}^{E}\left(e_{i}\right)}^{N-}(u)\right\} \leq\left\{N_{(N C)_{S 1}^{E}\left(e_{i}\right)}(u) \wedge N_{(N C))_{S 2}^{E}\left(e_{i}\right)}(u)\right\}
$$

for all $u \in U$ and $(g, z) \in\left(E_{1} \cap E_{2} \times X_{1} \cap X_{2}\right)$. Then $\left((N C)_{S 1}^{E}, E_{1}, X_{1}\right) \cup_{R}\left((N C)_{S 2}^{E}, E_{2}, X_{2}\right)$ is INCSESs in $U$.

Proof. By Definition 20, we know $\left((N C)_{S 1}^{E}, E_{1}, X_{1}\right) \cup_{R}\left((N C)_{S 2}^{E}, E_{2}, X_{2}\right)=\left((N C)_{S 3}^{E}, E_{3}, X_{3}\right)$, where $E_{3}=E_{1} \cup E_{2}, X_{3}=X_{1} \cup X_{2}$,

$$
(N C)_{S 3}^{E}\left(e_{3}, x_{3}\right)=\left\{\begin{array}{cl}
(N C)_{S 1}^{E}\left(e_{3}, x_{3}\right) & \text { if }\left(e_{3}, x_{3}\right) \in\left(E_{1} \times X_{1}\right)-\left(E_{2} \times X_{2}\right) \\
(N C)_{S 2}^{E}\left(e_{3}, x_{3}\right) & \text { if }\left(e_{3}, x_{3}\right) \in\left(E_{2} \times X_{2}\right)-\left(E_{1} \times X_{1}\right) \\
(N C)_{S 1}^{E}\left(e_{3}, x_{3}\right) \vee_{R}(N C)_{S 2}^{E}\left(e_{3}, x_{3}\right) & \text { if }\left(e_{3}, x_{3}\right) \in\left(E_{1} \times E_{2}\right) \cap\left(X_{1} \times X_{2}\right)
\end{array}\right.
$$

where

$$
\begin{aligned}
& (N C)_{S 1}^{E}\left(e_{3}, x_{3}\right) \vee_{R}(N C)_{S 2}^{E}\left(e_{3}, x_{3}\right)=\left\{\left\langle u, I_{(N C))_{S 1}^{E}\left(e_{3}, x_{3}\right)}^{N} \vee I_{(N C))_{S 2}^{E}\left(e_{3}, x_{3}\right)}^{N}, N_{(N C))_{S 1}^{E}\left(e_{3}, x_{3}\right)} \wedge N_{(N C))_{S 2}^{E}\left(e_{3}, x_{3}\right)}\right\rangle: u \in U\right\}
\end{aligned}
$$

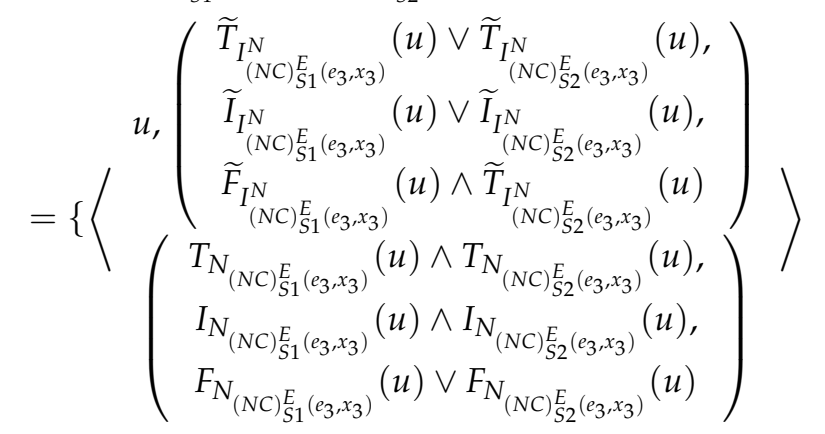

If $\left(e_{3}, x_{3}\right) \in\left(E_{1} \times X_{1}\right)-\left(E_{2} \times X_{2}\right)$ or if $\left(e_{3}, x_{3}\right) \in\left(E_{2} \times X_{2}\right)-\left(E_{1} \times X_{1}\right)$ then the result is trivial. If $\left(e_{3}, x_{3}\right) \in\left(E_{1} \cap E_{2} \times X_{1} \cap X_{2}\right)$, then

$$
(N C)_{S 3}^{E}\left(e_{3}, x_{3}\right)=\left\{\left\langle u, I_{(N C))_{S 1}^{E}\left(e_{3}, x_{3}\right)}^{N} \vee I_{(N C))_{S 2}^{E}\left(e_{3}, x_{3}\right)}^{N}, N_{(N C) S_{S 1}^{E}\left(e_{3}, x_{3}\right)} \wedge N_{(N C))_{S 2}^{E}\left(e_{3}, x_{3}\right)}\right\rangle: u \in U\right\} .
$$

Since $\left((N C)_{S 1}^{E}, E_{1}, X_{1}\right)$ and $\left((N C)_{S 2}^{E}, E_{2}, X_{2}\right)$ are INCSESs in $U$. So $I_{(N C)_{S 1}^{E}\left(e_{3}, x_{3}\right)}^{N-}(u) \leq$ $N_{(N C))_{S 1}^{E}\left(e_{3}, x_{3}\right)}(u) \leq I_{(N C))_{S 1}^{E}\left(e_{3}, x_{3}\right)}^{N+}(u)$ and $I_{(N C)_{S 2}^{E}\left(e_{3}, x_{3}\right)}^{N-}(u) \leq N_{(N C)_{S 2}^{E}\left(e_{3}, x_{3}\right)}(u) \leq I_{(N C)_{S 2}^{E}\left(e_{3}, x_{3}\right)}^{N+}(u)$. Also

$$
\begin{aligned}
I_{(N C)_{S 1}^{E}\left(e_{3}, x_{3}\right)}^{N-}(u) \vee I_{(N C)_{S 2}^{E}\left(e_{3}, x_{3}\right)}^{N-}(u) & \leq N_{(N C)_{S 1}^{E}\left(e_{3}, x_{3}\right)}(u) \wedge N_{(N C)_{S 2}^{E}\left(e_{3}, x_{3}\right)}(u) \\
& \leq I_{\left.(N C)_{S 1}^{N}+e_{3}, x_{3}\right)}^{N+}(u) \vee I_{(N C)_{S 2}^{E}\left(e_{3}, x_{3}\right)}^{N+}(u)
\end{aligned}
$$

for all $u \in U$ and $\left(e_{3}, x_{3}\right) \in\left(E_{2} \cap E_{2}, X_{1} \cap X_{2}\right)$. Hence $\left((N C)_{S 1}^{E}, E_{1}, X_{1}\right) \cup_{R}\left((N C)_{S 2}^{E}, E_{2}, X_{2}\right)$ is INCSESS in $U$.

2. The following example yields that $R$-intersection of two INCSESS need not be a INCSESS.

Example 5. Let $\left((N C)_{S 1}^{E}, E_{1}, X_{1}\right)$ and $\left((N C)_{S 2}^{E}, E_{2}, X_{2}\right)$ be two INCSESS in $U$, where 


$$
\left((N C)_{S 1}^{E}, E_{1}, X_{1}\right)=\left\{I_{(N C))_{S 1}^{N}\left(e_{i}\right)}^{N}=([0.1,0.2],[0.3,0.5],[0.3,0.6]), N_{(N C))_{S 1}^{E}\left(e_{i}\right)}=(0.2,0.3,0.4)\right\}
$$

and

$$
\left.\left((N C)_{S 2}^{E}, E_{2}, X_{2}\right)=\left\{I_{(N C))_{S 2}^{E}\left(e_{i}\right)}^{N}=([0.2,0.6],[0.3,0.6],[0.5,0.7]), N_{(N C)}^{E}\right)_{S 2}\left(e_{i}\right)=(0.4,0.6,0.5)\right\} .
$$

Now by Definition 20, we have $\left((N C)_{S 1}^{E}, E_{1}, X_{1}\right) \cap_{R}\left((N C)_{S 2}^{E}, E_{2}, X_{2}\right)=\left((N C)_{S 3}^{E}, E_{3}, X_{3}\right)$

$$
\left((N C)_{S 2}^{E}, E_{2}, X_{2}\right)=\left\{I_{(N C))_{S 3}^{N}\left(e_{i}\right)}^{N}=\left([0.1,0.2],[0.3,0.5],[0.5,0.7], N_{(N C))_{S \ni}^{E}(e i)}=(0.4,0.6,0.4)\right\} .\right.
$$

As $0.4 \notin[0.1,0.2], 0.6 \notin[0.3,0.5]$ and $0.4 \notin[0.5,0.7]$.

Hence $\left((N C)_{S 1}^{E}, E_{1}, X_{1}\right) \cap_{R}\left((N C)_{S 2}^{E}, E_{2}, X_{2}\right)$ is not a INCSES in $U$.

The following theorem gives the condition that $R$-intersection of two INCSESS is to be a INCSES.

Theorem 3. Let $\left((N C)_{S 1}^{E}, E_{1}, X_{1}\right)$ and $\left((N C)_{S 2}^{E}, E_{2}, X_{2}\right)$ be two INCSESs in $U$, and

$$
\text { where }\left((N C)_{S 1}^{E}, E_{1}, X_{1}\right)=\left\{(N C)_{S 1}^{E}(e, x)=\left\{\left\langle u, I_{1(e, x)}^{N}(u), N_{1(e, x)}(u)\right\rangle, u \in U\right\},(e, x) \in E_{1} \times X_{1}\right\}
$$
that

$$
\left((N C)_{S 2}^{E}, E_{2}, X_{2}\right)=\left\{(N C)_{S 2}^{E}(f, y)=\left\{\left\langle u, I_{2(f, y)}^{N}(u), N_{2(f, y)}(u)\right\rangle, u \in U\right\},(f, y) \in E_{2} \times X_{2}\right\} \text { such }
$$

$$
\inf \left\{I_{(N C))_{S}^{N+}\left(e_{i}\right)}^{N+}(x), I_{(N C))_{S 2}^{E}\left(e_{i}\right)}^{N+}(u)\right\} \geq\left\{N_{(N C))_{S}^{E}\left(e_{i}\right)}(u) \vee N_{(N C)_{S 2}^{E}\left(e_{i}\right)}(u)\right\}
$$

for all $u \in U$ and $(g, z) \in\left(E_{1} \cap E_{2} \times X_{1} \cap X_{2}\right)$. Then $\left((N C)_{S 1}^{E}, E_{1}, X_{1}\right) \cap_{R}\left((N C)_{S 2}^{E}, E_{2}, X_{2}\right)$ is a INCSES in $U$.

Proof. Similar to the proof of the Theorem 2.

\section{The following example yields that R-union of two ENCSESS need not be an ENCSESS.}

Example 6. Let $\left((N C)_{S 1}^{E}, E_{1}, X_{1}\right)$ and $\left((N C)_{S 2}^{E}, E_{2}, X_{2}\right)$ be two ENCSESs in $U$, where

$$
\left((N C)_{S 1}^{E}, E_{1}, X_{1}\right)=\left\{I_{(N C))_{S 1}^{E}\left(e_{i}\right)}^{N}=([0.3,0.4],[0.4,0.7],[0.3,0.6]), N_{(N C) E}^{E}\left(e_{i}\right)=(0.5,0.3,0.7)\right\}
$$

and

$$
\left((N C)_{S 2}^{E}, E_{2}, X_{2}\right)=\left\{I_{(N C))_{S 2}^{E}\left(e_{i}\right)}^{N}=([0.2,0.6],[0.3,0.5],[0.5,0.6]), N_{(N C))_{S 2}^{E}\left(e_{i}\right)}=(0.1,0.2,0.4)\right\} .
$$

Now by Definition 20, we have $\left((N C)_{S 1}^{E}, E_{1}, X_{1}\right) \cup_{R}\left((N C)_{S 2}^{E}, E_{2}, X_{2}\right)=\left((N C)_{S 3}^{E}, E_{3}, X_{3}\right)$

$$
\left((N C)_{S 3}^{E}, E_{3}, X_{3}\right)=\left\{I_{(N C))_{S 3}^{N}\left(e_{i}\right)}^{N}=\left([0.3,0.6],[0.4,0.7],[0.5,0.6], N_{(N C))_{S 3}^{E}(e i)}=(0.1,0.2,0.7)\right\} .\right.
$$

As $0.1 \notin[0.3,0.6], 0.2 \notin[0.4,0.7]$ and $0.7 \notin[0.5,0.6]$.

Hence $R$-union is not a ENCSESS in $U$.

The following theorem gives the condition that R-union of two ENCSESS to be a ENCSESs.

Theorem 4. Let $\left((N C)_{S 1}^{E}, E_{1}, X_{1}\right)$ and $\left((N C)_{S 2}^{E}, E_{2}, X_{2}\right)$ be two ENCSESs in $U$, and

where $\left((N C)_{S 1}^{E}, E_{1}, X_{1}\right)=\left\{(N C)_{S 1}^{E}(e, x)=\left\{\left\langle u, I_{1(e, x)}^{N}(u), N_{1(e, x)}(u)\right\rangle, u \in U\right\},(e, x) \in E_{1} \times X_{1}\right\}$ 
such that

$$
\left((N C)_{S 2}^{E}, E_{2}, X_{2}\right)=\left\{(N C)_{S 2}^{E}(f, y)=\left\{\left\langle u, I_{2(f, y)}^{N}(u), N_{2(f, y)}(u)\right\rangle, u \in U\right\},(f, y) \in E_{2} \times X_{2}\right\}
$$

$$
\begin{aligned}
& \left\{\inf \left\{\sup \left\{I_{(N C))_{S 1}^{E}\left(e_{i}\right)}^{N+}(u), I_{(N C)_{S 2}^{E}\left(e_{i}\right)}^{N-}(u)\right\}, \sup I_{(N C)_{S 1}^{E}\left(e_{i}\right)}^{N-}(u), I_{(N C))_{S 2}^{E}\left(e_{i}\right)}^{N+}(u)\right\}\right\} \\
& <\left\{N_{(N C))_{S 1}^{E}\left(e_{i}\right)}(u) \wedge N_{(N C))_{S 2}^{E}\left(e_{i}\right)}(u)\right\} \\
& \left.\leq\left\{\sup \left\{I_{(N C))_{S 1}^{N+}\left(e_{i}\right)}^{N+}(u), I_{(N C)_{S 2}^{E}\left(e_{i}\right)}^{N-}(u)\right\}, \sup I_{(N C)_{S 1}^{E}\left(e_{i}\right)}^{N-}(u), I_{(N C))_{S 2}^{E}\left(e_{i}\right)}^{N+}(u)\right\}\right\},
\end{aligned}
$$

for all $u \in U$ and $(g, z) \in\left(E_{1} \cap E_{2} \times X_{1} \cap X_{2}\right)$. Then $\left((N C)_{S 1}^{E}, E_{1}, X_{1}\right) \cup_{R}\left((N C)_{S 2}^{E}, E_{2}, X_{2}\right)$ is a ENCSES in $U$.

Proof. By Definition 20, we know $\left((N C)_{S 1}^{E}, E_{1}, X_{1}\right) \cup_{R}\left((N C)_{S 2}^{E}, E_{2}, X_{2}\right)=\left((N C)_{S 3^{2}}^{E}, E_{3}, X_{3}\right)$, where $E_{3}=E_{1} \cup E_{2}, X_{3}=X_{1} \cup X_{2}$,

$$
(N C)_{S 3}^{E}\left(e_{3}, x_{3}\right)=\left\{\begin{array}{cl}
(N C)_{S 1}^{E}\left(e_{3}, x_{3}\right) & \text { if }\left(e_{3}, x_{3}\right) \in\left(E_{1} \times X_{1}\right)-\left(E_{2} \times X_{2}\right) \\
(N C)_{S 2}^{E}\left(e_{3}, x_{3}\right) & \text { if }\left(e_{3}, x_{3}\right) \in\left(E_{2} \times X_{2}\right)-\left(E_{1} \times X_{1}\right) \\
(N C)_{S 1}^{E}\left(e_{3}, x_{3}\right) \vee_{R}(N C)_{S 2}^{E}\left(e_{3}, x_{3}\right) & \text { if }\left(e_{3}, x_{3}\right) \in\left(E_{1} \times E_{2}\right) \cap\left(X_{1} \times X_{2}\right)
\end{array}\right.
$$

where

$$
\begin{aligned}
& (N C)_{S 1}^{E}\left(e_{3}, x_{3}\right) \vee_{R}(N C)_{S 2}^{E}\left(e_{3}, x_{3}\right)=\left\{\left\langle\begin{array}{c}
u, I_{(N C))_{S 1}^{E}\left(e_{3}, x_{3}\right)}^{N} \vee I_{(N C)_{S 2}^{E}\left(e_{3}, x_{3}\right)^{\prime}}^{N} N_{(N C)_{S 1}^{E}\left(e_{3}, x_{3}\right)} \wedge N_{(N C)_{S 2}^{E}\left(e_{3}, x_{3}\right)}
\end{array}\right\rangle: u \in U\right\}
\end{aligned}
$$

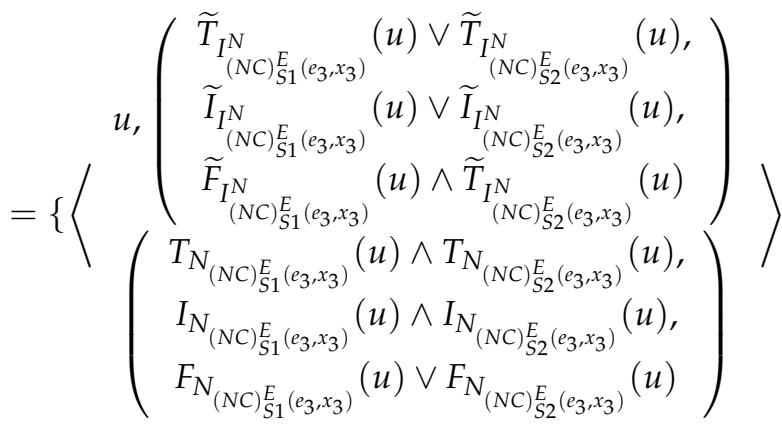

If $\left(e_{3}, x_{3}\right) \in\left(E_{1} \cap E_{2} \times X_{1} \cap X_{2}\right)$, take

$$
h=\left(\begin{array}{c}
\left\{\operatorname { i n f } \left\{\sup \left\{I_{(N C))_{S 1}^{N+}\left(e_{i}\right)}^{N+}(u), I_{(N C))_{S 2}^{E}\left(e_{i}\right)}^{N-}(u)\right\}\right.\right. \\
\left.\sup \left\{I_{(N C))_{S 1}^{E}\left(e_{i}\right)}^{N-}(u), I_{(N C))_{S 2}^{N+}\left(e_{i}\right)}^{N+}(u)\right\}\right\}
\end{array}\right)
$$

and

$$
k=\left(\begin{array}{c}
\left\{\sup \left\{I_{(N C))_{S 1}^{E+}\left(e_{i}\right)}^{N+}(u), I_{(N C))_{S 2}^{E}\left(e_{i}\right)}^{N-}(u)\right\}\right. \\
\left.\left.\sup I_{(N C))_{S 1}^{E}\left(e_{i}\right)}^{N-}(u), I_{(N C))_{S 2}^{N+}\left(e_{i}\right)}^{N}(u)\right\}\right\}
\end{array}\right)
$$

Then $h$ is one of

$$
\left(I_{(N C))_{S 1}^{E}\left(e_{i}\right)}^{N+}(u), I_{(N C))_{S 2}^{E}\left(e_{i}\right)}^{N-}(u), I_{(N C))_{S 1}^{N-}\left(e_{i}\right)}^{N-}(u), I_{(N C))_{S 2}^{E}\left(e_{i}\right)}^{N+}(u)\right) .
$$

If we choose $h=I_{(N C))_{S 2}^{N-}\left(e_{i}\right)}^{N-}(u)$ or $I_{(N C))_{S 2}^{E}\left(e_{i}\right)}^{N+}(u)$, then

$$
I_{(N C)_{S 1}^{E}\left(e_{i}\right)}^{N-}(u) \leq I_{(N C))_{S 1}^{E}\left(e_{i}\right)}^{N+}(u) \leq I_{(N C)_{S 2}^{E}\left(e_{i}\right)}^{N-}(u) \leq I_{(N C)_{S 2}^{E}\left(e_{i}\right)}^{N+}(u)
$$


and so $k=I_{(N C))_{S 1}^{E}\left(e_{i}\right)}^{N+}(u)$. Thus

$$
\left(\begin{array}{c}
\sup \left\{I_{(N C))_{S 1}^{E}\left(e_{i}\right)}^{N-}(u), I_{(N C))_{S 2}^{E}\left(e_{i}\right)}^{N-}(u)\right\}=I_{(N C))_{S 2}^{E}\left(e_{i}\right)}^{N-}(u) \\
=h>\left\{N_{(N C)_{S 1}^{E}\left(e_{i}\right)}(u) \wedge N_{(N C)_{S 2}^{E}\left(e_{i}\right)}(u)\right\}
\end{array}\right) .
$$

Hence

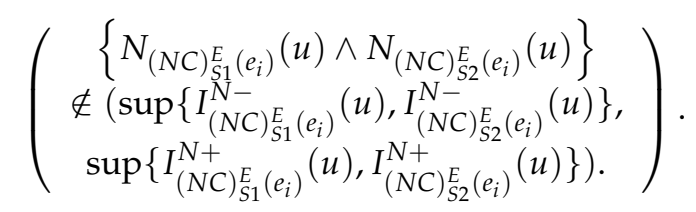

Now if $h=I_{(N C))_{S 2}^{E}\left(e_{i}\right)}^{N+}(u)$ then $I_{(N C)_{S 1}^{E}\left(e_{i}\right)}^{N-}(u) \leq I_{(N C)_{S 2}^{E}\left(e_{i}\right)}^{N+}(u) \leq I_{(N C))_{S 1}^{E}\left(e_{i}\right)}^{N+}(u)$ and so $\sup \left\{I_{(N C)_{S 1}^{E}\left(e_{i}\right)}^{N-}(u), I_{(N C))_{S 2}^{N-}\left(e_{i}\right)}^{N-}(u)\right\}$. Assume $k=I_{(N C))_{S 1}^{E}\left(e_{i}\right)}^{N-}(u)$, then we have

$$
\begin{aligned}
I_{(N C))_{S 2}^{E}\left(e_{i}\right)}^{N-}(u) & \leq I_{(N C))_{S 1}^{E}\left(e_{i}\right)}^{N-}(u)<\left\{N_{(N C))_{S 1}^{E}\left(e_{i}\right)}(u) \wedge N_{(N C))_{S 2}^{E}\left(e_{i}\right)}(u)\right\} \\
& <I_{(N C))_{S 2}^{E}\left(e_{i}\right)}^{N+}(u) \leq I_{(N C))_{S 1}^{E}\left(e_{i}\right)}^{N+}(u) .
\end{aligned}
$$

So, we can write

$$
\left(\begin{array}{c}
I_{(N C))_{S 2}^{E}\left(e_{i}\right)}^{N-}(u) \leq I_{(N C))_{S 1}^{E}\left(e_{i}\right)}^{N-}(u)< \\
\left\{N_{(N C)_{S 1}^{E}\left(e_{i}\right)}(u) \wedge N_{(N C))_{S 2}^{E}\left(e_{i}\right)}(u)\right\} \\
=I_{(N C))_{S 2}^{E+}\left(e_{i}\right)}^{N+}(u) \leq I_{(N C))_{S 1}^{E}\left(e_{i}\right)}^{N+}(u)
\end{array}\right)
$$

For the case

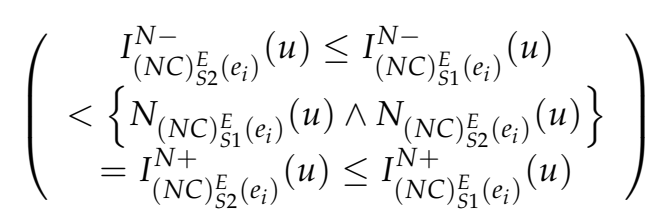

which contradicted the fact that $\left((N C)_{S 1}^{E}, E_{1}, X_{1}\right)$ and $\left((N C)_{S 2}^{E}, E_{2}, X_{2}\right)$ be two ENCSESs in $U$. For the case

$$
\left(\begin{array}{c}
I_{(N C)_{S 2}^{E}\left(e_{i}\right)}^{N-}(u) \leq I_{(N C))_{S 1}^{E}\left(e_{i}\right)}^{N-}(u)= \\
\left\{N_{(N C))_{S 1}^{E}\left(e_{i}\right)}(u) \wedge N_{(N C))_{S 2}^{E}\left(e_{i}\right)}(u)\right\} \\
<I_{(N C)_{S 2}^{N+}\left(e_{i}\right)}(u) \leq I_{(N C))_{S 1}^{E}\left(e_{i}\right)}^{N+}(u)
\end{array}\right)
$$

we have

$$
\left(\begin{array}{c}
\left\{N_{(N C) S_{S 1}^{E}\left(e_{i}\right)}(u) \wedge N_{(N C))_{S 2}^{E}\left(e_{i}\right)}(u)\right\} \\
\notin\left(\sup \left\{I_{(N C))_{S 1}^{N}\left(e_{i}\right)}^{N}(u), I_{(N C)_{S 2}^{E}\left(e_{i}\right)}^{N}(u)\right\}\right) \\
=I_{(N C))_{S 1}^{E}\left(e_{i}\right)}^{N-}(u)=\left\{N_{(N C))_{S 1}^{E}\left(e_{i}\right)}(u) \wedge N_{(N C))_{S 2}^{E}\left(e_{i}\right)}(u)\right\}
\end{array}\right) .
$$

Again, assume that $k=I_{(N C))_{S 2}^{N-}\left(e_{i}\right)}^{N-}(u)$, then we have

$$
\begin{aligned}
I_{(N C))_{S 1}^{E}\left(e_{i}\right)}^{N-}(u) & \leq I_{(N C))_{S 2}^{E}\left(e_{i}\right)}^{N-}(u)<\left\{N_{(N C))_{S 1}^{E}\left(e_{i}\right)}(u) \wedge N_{(N C))_{S 2}^{E}\left(e_{i}\right)}(u)\right\} \\
& <I_{(N C))_{S 2}^{E}\left(e_{i}\right)}^{N+}(u) \leq I_{(N C))_{S 1}^{E}\left(e_{i}\right)}^{N+}(u)
\end{aligned}
$$


or

$$
\begin{aligned}
I_{(N C))_{S 1}^{E}\left(e_{i}\right)}^{N-}(u) & \leq I_{(N C))_{S 2}^{E}\left(e_{i}\right)}^{N-}(u)=\left\{N_{(N C)_{S 1}^{E}\left(e_{i}\right)}(u) \wedge N_{(N C)_{S 2}^{E}\left(e_{i}\right)}(u)\right\} \\
& <I_{(N C))_{S 2}^{E}\left(e_{i}\right)}^{N+}(u) \leq I_{(N C))_{S 1}^{E}\left(e_{i}\right)}^{N+}(u) .
\end{aligned}
$$

For the case

$$
\begin{aligned}
I_{(N C)_{S 1}^{E}\left(e_{i}\right)}^{N-}(u) & \leq I_{(N C))_{S 2}^{E}\left(e_{i}\right)}^{N-}(u)<\left\{N_{(N C))_{S 1}^{E}\left(e_{i}\right)}(u) \wedge N_{(N C)_{S 2}^{E}\left(e_{i}\right)}(u)\right\} \\
& <I_{(N C)_{S 2}^{E}\left(e_{i}\right)}^{N+}(u) \leq I_{(N C))_{S 1}^{E}\left(e_{i}\right)}^{N+}(u)
\end{aligned}
$$

which contradict $\left((N C)_{S 1}^{E}, E_{1}, X_{1}\right)$ and $\left((N C)_{S 2}^{E}, E_{2}, X_{2}\right)$ be two ENCSESs in $U$. For the case

$$
\begin{aligned}
& I_{(N C))_{S 1}^{E}\left(e_{i}\right)}^{N-}(u) \leq I_{(N C))_{S 2}^{E}\left(e_{i}\right)}^{N-}(u)=\left\{N_{(N C))_{S 1}^{E}\left(e_{i}\right)}(u) \wedge N_{(N C)_{S 2}^{E}\left(e_{i}\right)}(u)\right\} \\
&<I_{(N C))_{S 2}^{E}\left(e_{i}\right)}^{N+}(u) \leq I_{(N C))_{S 1}^{E}\left(e_{i}\right)}^{N+}(u)
\end{aligned}
$$

we have

$$
\begin{aligned}
\left\{N_{(N C) S_{S 1}^{E}\left(e_{i}\right)}(u) \wedge N_{(N C) S_{S 2}^{E}\left(e_{i}\right)}(u)\right\} & \notin\left\{\left(\left(\sup \left\{I_{(N C))_{S 1}^{N-}\left(e_{i}\right)}^{N-}(u), I_{(N C))_{S 2}^{E}\left(e_{i}\right)}^{N-}(u)\right\}\right),\right.\right. \\
& \left.\left(\sup \left\{I_{(N C))_{S 1}^{N+}\left(e_{i}\right)}^{N+}(u), I_{(N C))_{S 2}^{E}\left(e_{i}\right)}^{N+}(u)\right\}\right)\right\}
\end{aligned}
$$

because

$$
\left(\left(\sup \left\{I_{(N C))_{S 1}^{E}\left(e_{i}\right)}^{N-}(u), I_{(N C))_{S 2}^{E}\left(e_{i}\right)}^{N-}(u)\right\}\right)=, I_{(N C))_{S 2}^{E}\left(e_{i}\right)}^{N-}(u)=\left\{N_{(N C))_{S 1}^{E}\left(e_{i}\right)}(u) \wedge N_{(N C)_{S 2}^{E}\left(e_{i}\right)}(u)\right\} .\right.
$$

If $e_{i}=\left(e_{3}, x_{3}\right) \in\left(E_{1} \times X_{1}\right)-\left(E_{2} \times X_{2}\right)$ or if $e_{i}=\left(e_{3}, x_{3}\right) \in\left(E_{2} \times X_{2}\right)-\left(E_{1} \times X_{1}\right)$ then the result is trivial. Hence $\left((N C)_{S 1}^{E}, E_{1}, X_{1}\right) \cup_{R}\left((N C)_{S 2}^{E}, E_{2}, X_{2}\right)$ is a ENCSES in $U$.

4. The following example shows that $R$-intersection of two ENCSESS need not be ENCSESs.

Example 7. Let $\left((N C)_{S 1}^{E}, E_{1}, X_{1}\right)$ and $\left((N C)_{S 2}^{E}, E_{2}, X_{2}\right)$ be two ENCSESs in $U$, where

$$
\left((N C)_{S 1}^{E}, E_{1}, X_{1}\right)=\left\{I_{(N C))_{S 1}^{N}\left(e_{i}\right)}^{N}=([0.3,0.4],[0.4,0.7],[0.5,0.6]), N_{(N C)_{S 1}^{E}\left(e_{i}\right)}=(0.2,0.3,0.4)\right\}
$$

and

$$
\left((N C)_{S 2}^{E}, E_{2}, X_{2}\right)=\left\{I_{(N C)_{S 2}^{E}\left(e_{i}\right)}^{N}=([0.2,0.3],[0.3,0.5],[0.6,0.7]), N_{(N C)_{S 2}^{E}\left(e_{i}\right)}=(0.4,0.6,0.5)\right\} .
$$

Now by Definition 20, we have $\left((N C)_{S 1}^{E}, E_{1}, X_{1}\right) \cap_{R}\left((N C)_{S 2}^{E}, E_{2}, X_{2}\right)=\left((N C)_{S 3}^{E}, E_{3}, X_{3}\right)$

$$
\left((N C)_{S 3}^{E}, E_{3}, X_{3}\right)=\left\{I_{(N C))_{S 3}^{E}\left(e_{i}\right)}^{N}=\left([0.2,0.3],[0.3,0.5],[0.6,0.7], N_{(N C))_{S 3}^{E}(e i)}=(0.4,0.6,0.4)\right\} .\right.
$$

As $0.4 \notin[0.2,0.3], 0.6 \notin[0.3,0.5]$ and $0.4 \notin[0.6,0.7]$. Hence $\left((N C)_{S 1}^{E}, E_{1}, X_{1}\right) \cap_{R}\left((N C)_{S 2}^{E}, E_{2}, X_{2}\right)$ is not a ENCSES in $U$.

The following theorem gives the condition that R-intersection of two ENCSESs is also a ENCSES.

Theorem 5. Let $\left((N C)_{S 1}^{E}, E_{1}, X_{1}\right)$ and $\left((N C)_{S 2}^{E}, E_{2}, X_{2}\right)$ be two ENCSESs in $U$, 

and

where $\left((N C)_{S 1}^{E}, E_{1}, X_{1}\right)=\left\{(N C)_{S 1}^{E}(e, x)=\left\{\left\langle u, I_{1(e, x)}^{N}(u), N_{1(e, x)}(u)\right\rangle, u \in U\right\},(e, x) \in E_{1} \times X_{1}\right\}$ such that

$\left((N C)_{S 2}^{E}, E_{2}, X_{2}\right)=\left\{(N C)_{S 2}^{E}(f, y)=\left\{\left\langle u, I_{2(f, y)}^{N}(u), N_{2(f, y)}(u)\right\rangle, u \in U\right\},(f, y) \in E_{2} \times X_{2}\right\}$

$$
\begin{aligned}
& \left\{\operatorname { i n f } \left\{\operatorname { s u p } \left\{I_{(N C) E}^{N+}\left(e_{i}\right)\right.\right.\right. \\
& \left.\left.<\left\{u, I_{(N C)_{S 2}^{E}\left(e_{i}\right)}^{N-}(u)\right\}, \sup I_{(N C)_{S}^{E}\left(e_{i}\right)}^{N-}(u), I_{(N C))_{S 2}^{E}\left(e_{i}\right)}^{N+}(u)\right\}\right\} \\
& \leq\left\{N_{(N C))_{S}^{E}\left(e_{i}\right)}(u) \vee N_{(N C))_{S 2}^{E}\left(e_{i}\right)}(u)\right\} \\
& \left.\left.\leq \sup \left\{I_{(N C))_{S}^{E}\left(e_{i}\right)}^{N+}(u), I_{(N C))_{S 2}^{E}\left(e_{i}\right)}^{--}(u)\right\}, \sup I_{(N C)_{S}^{E}\left(e_{i}\right)}^{N-}(u), I_{(N C))_{S 2}^{E}\left(e_{i}\right)}^{N+}(u)\right\}\right\}
\end{aligned}
$$

for all $u \in U$ and $(g, z) \in\left(E_{1} \cap E_{2} \times X_{1} \cap X_{2}\right)$. Then $\left((N C)_{S 1}^{E}, E_{1}, X_{1}\right) \cap_{R}\left((N C)_{S 2}^{E}, E_{2}, X_{2}\right)$ is ENCSESs in $U$.

Proof. Similar to the proof of Theorem 4.

\section{The following example shows that $P$-union of two ENCSESS need not to be a ENCSES.}

Example 8. Let $\left((N C)_{S 1}^{E}, E_{1}, X_{1}\right)$ and $\left((N C)_{S 2}^{E}, E_{2}, X_{2}\right)$ be two ENCSESs in $U$, where

$$
\left((N C)_{S 1}^{E}, E_{1}, X_{1}\right)=\left\{I_{(N C))_{S 1}^{E}\left(e_{i}\right)}^{N}=([0.3,0.5],[0.4,0.7],[0.5,0.6]), N_{(N C))_{S 1}^{E}\left(e_{i}\right)}=(0.6,0.8,0.4)\right\}
$$

and

$$
\left.((N C))_{S 2}^{E}, E_{2}, X_{2}\right)=\left\{I_{(N C)_{S 2}^{E}\left(e_{i}\right)}^{N}=([0.2,0.3],[0.3,0.5],[0.6,0.7]), N_{(N C))_{S 2}^{E}\left(e_{i}\right)}=(0.4,0.6,0.5)\right\} .
$$

Now by Definition 20, we have

$$
\left((N C)_{S 3}^{E}, E_{3}, X_{3}\right)=\left\{I_{(N C))_{S 3}^{E}\left(e_{i}\right)}^{N}=\left([0.3,0.5],[0.4,0.7],[0.5,0.6], N_{(N C))_{S 3}^{E}(e i)}=(0.6,0.8,0.4)\right\} .\right.
$$

As $0.6 \notin[0.3,0.5], 0.8 \notin[0.4,0.7]$ and $0.4 \notin[0.5,0.6]$.

Hence P-union of $\left((N C)_{S 1}^{E}, E_{1}, X_{1}\right)$ and $\left((N C)_{S 2}^{E}, E_{2}, X_{2}\right)$ is not ENCSES in $U$.

The following theorem gives the condition under which P-union of two ENCSESS is a ENCSESs.

Theorem 6. Let $\left((N C)_{S 1}^{E}, E_{1}, X_{1}\right)$ and $\left((N C)_{S 2}^{E}, E_{2}, X_{2}\right)$ be two ENCSESs in $U$, and

where $\left((N C)_{S 1}^{E}, E_{1}, X_{1}\right)=\left\{(N C)_{S 1}^{E}(e, x)=\left\{\left\langle u, I_{1(e, x)}^{N}(u), N_{1(e, x)}(u)\right\rangle, u \in U\right\},(e, x) \in E_{1} \times X_{1}\right\}$ such that

$$
\left((N C)_{S 2}^{E}, E_{2}, X_{2}\right)=\left\{(N C)_{S 2}^{E}(f, y)=\left\{\left\langle u, I_{2(f, y)}^{N}(u), N_{2(f, y)}(u)\right\rangle, u \in U\right\},(f, y) \in E_{2} \times X_{2}\right\}
$$

$$
\begin{aligned}
& \sup \left\{\inf \left\{I_{(N C))_{S}^{E}\left(e_{i}\right)}^{N+}(u), I_{(N C)_{S 2}^{E}\left(e_{i}\right)}^{N-}(u)\right\},\left\{I_{(N C))_{S}^{E}\left(e_{i}\right)}^{N-}(u), I_{(N C)_{S 2}^{E}\left(e_{i}\right)}^{N+}(u)\right\}\right\} \\
& <\left\{N_{(N C))_{S}^{E}\left(e_{i}\right)}(u) \vee N_{(N C)_{S 2}^{E}\left(e_{i}\right)}(u)\right\} \\
& \left.\leq \inf \left\{\sup \left\{I_{(N C)_{S}^{E}\left(e_{i}\right)}^{N+}(u), I_{(N C)_{S 2}^{E}\left(e_{i}\right)}^{N-}(u)\right\}, \sup I_{(N C))_{S}^{E}\left(e_{i}\right)}^{N-}(u), I_{(N C))_{S 2}^{E}\left(e_{i}\right)}^{N+}(u)\right\}\right\}
\end{aligned}
$$

for all $e \in(A \cap A \times B \cap B)$ and $u \in U$. Then $\left((N C)_{S 1}^{E}, E_{1}, X_{1}\right) \cup_{P}\left((N C)_{S 2}^{E}, E_{2}, X_{2}\right)$ is a ENCSES over $U$.

Proof. Similar to the proof of Theorems 2 and 4.

6. The following example shows that $P$-intersection of two ENCSESS need not be a ENCSES. 
Example 9. Let $\left((N C)_{S 1}^{E}, E_{1}, X_{1}\right)$ and $\left((N C)_{S 2}^{E}, E_{2}, X_{2}\right)$ be two ENCSESs in $U$, where

$$
\left((N C)_{S 1}^{E}, E_{1}, X_{1}\right)=\left\{I_{(N C))_{S 1}^{E}\left(e_{i}\right)}^{N}=([0.3,0.5],[0.4,0.7],[0.5,0.6]), N_{(N C))_{S 1}^{E}\left(e_{i}\right)}=(0.6,0.8,0.4)\right\}
$$

and

$$
\left((N C)_{S 2}^{E}, E_{2}, X_{2}\right)=\left\{I_{(N C))_{S 2}^{E}\left(e_{i}\right)}^{N}=([0.2,0.3],[0.3,0.5],[0.6,0.7]), N_{(N C))_{S 2}^{E}\left(e_{i}\right)}=(0.4,0.6,0.5)\right\} .
$$

Now by Definition 20, we have

$$
\left((N C)_{S 3}^{E}, E_{3}, X_{3}\right)=\left\{I_{(N C))_{S 3}^{E}\left(e_{i}\right)}^{N}=\left([0.2,0.3],[0.3,0.5],[0.6,0.7], N_{(N C))_{S 3}^{E}(e i)}=(0.4,0.6,0.5)\right\} .\right.
$$

As $0.4 \notin[0.2,0.3], 0.6 \notin[0.3,0.5]$ and $0.5 \notin[0.6,0.7]$.

Hence P-intersection of $\left((N C)_{S 1}^{E}, E_{1}, X_{1}\right)$ and $\left((N C)_{S 2}^{E}, E_{2}, X_{2}\right)$ is not ENCSES in $U$.

Theorem 7. Let $\left\{(N C)_{S i}^{E}\right\}_{i \in I}=\left\{\left((N C)_{S i}^{E}, E_{i}, X_{i}\right)\right\}_{i \in I}$ be a family of internal cubic soft expert set (INCSESs) sets over $U, u \in U$ for any $\left.e_{i} \in E_{i}, x \in X_{i}\right\}$. Then $\cup_{P_{i \in I}}\left\{(N C)_{S i}^{E}\right\}$ and $\cap_{P_{i \in I}}\left\{(N C)_{\text {Si }}^{E}\right\}$ are internal cubic soft expert set (INCSESS) sets over $U$.

Proof. As $\left\{(N C)_{S i}^{E}\right\}_{i \in I}$ be a family of internal generalized cubic soft expert set (INCSESs) over $U$ so we have $I_{(N C)_{S i}^{E}(e, x)}^{N-}(u) \leq N_{(N C)_{S i}^{E}(e, x)}(u) \leq I_{(N C))_{S i}^{E}(e, x)}^{N-}(u)$ for each $i \in I$ this implies that

$$
\cup_{P_{i \in I}} I_{(N C))_{S i}^{N}(e, x)}^{N-}(u) \leq \cup_{P_{i \in I}} N_{(N C))_{S i}^{E}(e, x)}(u) \leq \cup_{P_{i \in I}} I_{(N C)_{S i}^{E}(e, x)}^{N-}(u)
$$

and

$$
\cap_{P_{i \in I}} I_{(N C))_{S i}^{E}(e, x)}^{N-}(u) \leq \cap_{P_{i \in I}} N_{(N C)_{S i}^{E}(e, x)}(u) \leq \cap_{P_{i \in I}} I_{(N C))_{S i}^{E}(e, x)}^{N-}(u) .
$$

Hence $\underset{i \in I}{\cup_{p}}\left\{(N C)_{S i}^{E}\right\}$ and $\cap_{i \in I}\left\{(N C)_{S i}^{E}\right\}$ are (INCSESs) over $U$.

Theorem 8. Let $\left((N C)_{S 1}^{E}, E_{1}, X_{1}\right)$ and $\left((N C)_{S 2}^{E}, E_{2}, X_{2}\right)$ be two ENCSESs in $U$,

$$
\text { where }\left((N C)_{S 1}^{E}, E_{1}, X_{1}\right)=\left\{(N C)_{S 1}^{E}(e, x)=\left\{\left\langle u, I_{1(e, x)}^{N}(u), N_{1(e, x)}(u)\right\rangle, u \in U\right\},(e, x) \in E_{1} \times X_{1}\right\}
$$
such that

and $\left((N C)_{S 2}^{E}, E_{2}, X_{2}\right)=\left\{(N C)_{S 2}^{E}(f, y)=\left\{\left\langle u, I_{2(f, y)}^{N}(u), N_{2(f, y)}(u)\right\rangle, u \in U\right\},(f, y) \in E_{2} \times X_{2}\right\}$

$$
\begin{aligned}
& \sup \left\{\inf \left\{I_{(N C))_{S}^{E}\left(e_{i}\right)}^{N+}(u), I_{(N C)_{S 2}^{E}\left(e_{i}\right)}^{N-}(u)\right\},\left\{I_{(N C)_{S}^{E}\left(e_{i}\right)}^{N-}(u), I_{(N C))_{S 2}^{E}\left(e_{i}\right)}^{N+}(u)\right\}\right\} \\
& <\left\{N_{(N C)_{S}^{E}\left(e_{i}\right)}(u) \wedge N_{(N C)_{S 2}^{E}\left(e_{i}\right)}(u)\right\} \\
& \left.\leq \inf \left\{\sup \left\{I_{(N C))_{S}^{E}\left(e_{i}\right)}^{N+}(u), I_{(N C)_{S 2}^{E}\left(e_{i}\right)}^{N-}(u)\right\}, \sup I_{(N C))_{S}^{E}\left(e_{i}\right)}^{N-}(u), I_{(N C))_{S 2}^{E}\left(e_{i}\right)}^{N+}(u)\right\}\right\}
\end{aligned}
$$

for all $e \in(A \cap A \times B \cap B)$ and $u \in U$. Then $\left((N C)_{S 1}^{E}, E_{1}, X_{1}\right) \cap_{P}\left((N C)_{S 2}^{E}, E_{2}, X_{2}\right)$ is also an ENCSESs and INCSESs over $U$.

Proof. Similar to the proof of Theorems 2 and 4.

Theorem 9. Let $\left((N C)_{S 1}^{E}, E_{1}, X_{1}\right)$ and $\left((N C)_{S 2}^{E}, E_{2}, X_{2}\right)$ be two ENCSESs in $U$, and where $\left((N C)_{S 1}^{E}, E_{1}, X_{1}\right)=\left\{(N C)_{S 1}^{E}(e, x)=\left\{\left\langle u, I_{1(e, x)}^{N}(u), N_{1(e, x)}(u)\right\rangle, u \in U\right\},(e, x) \in E_{1} \times X_{1}\right\}$ 

that

$\left((N C)_{S 2}^{E}, E_{2}, X_{2}\right)=\left\{(N C)_{S 2}^{E}(f, y)=\left\{\left\langle u, I_{2(f, y)}^{N}(u), N_{2(f, y)}(u)\right\rangle, u \in U\right\},(f, y) \in E_{2} \times X_{2}\right\}$ such $\left(N_{(N C))_{S}^{E}\left(e_{i} x\right)} \vee N_{(N C))_{S 2}^{E}\left(e_{i}\right)}\right)(u) \in\left\{\begin{array}{c}\inf \left\{\sup \left\{I_{(N C))_{S}^{E}\left(e_{i}\right)}^{N+}(u), I_{(N C))_{S 2}^{N-}\left(e_{i}\right)}^{N-}(u)\right\}, \sup \left\{I_{(N C))_{S}^{E}\left(e_{i}\right)}^{N-}(u), I_{(N C))_{S 2}^{N}\left(e_{i}\right)}^{N+}(u)\right\}\right\}, \\ \quad\left\{\inf \left\{I_{(N C))_{S}^{N+}\left(e_{i}\right)}^{N+}(u), I_{(N C))_{S 2}^{N-}\left(e_{i}\right)}^{N-}(u)\right\}, \inf \left\{I_{(N C))_{S}^{N-}\left(e_{i}\right)}^{N-}(u), I_{(N C))_{S 2}^{N+}\left(e_{i}\right)}^{N+}(u)\right\}\right\}\end{array}\right.$ for all $e \in(A \cap A \times B \cap B)$ and $u \in U$. Then $\left((N C)_{S 1}^{E}, E_{1}, X_{1}\right) \cap_{R}\left((N C)_{S 2}^{E}, E_{2}, X_{2}\right)$ is a ENCSES and INCSES over $U$.

Proof. Similar to the proof of Theorems 2 and 4.

Theorem 10. Let $\left((N C)_{S 1}^{E}, E_{1}, X_{1}\right)$, and $\left((N C)_{S 2}^{E}, E_{2}, X_{2}\right)$, be any two NCSESs in $U$. Then (i) $\left((N C)_{S 1}^{E}, E_{1}, X_{1}\right) \cup_{P}\left(\left((N C)_{S 1}^{E}, E_{1}, X_{1}\right) \cap_{P}\left((N C)_{S 2}^{E}, E_{2}, X_{2}\right)\right)=\left((N C)_{S 1}^{E}, E_{1}, X_{1}\right)$, (ii) $\left((N C)_{S 1}^{E}, E_{1}, X_{1}\right) \cap_{P}\left(\left((N C)_{S 2}^{E}, E_{2}, X_{2}\right) \cup_{P}\left((N C)_{S 2}^{E}, E_{2}, X_{2}\right)\right)=\left((N C)_{S 1}^{E}, E_{1}, X_{1}\right)$, (iii) $\left(\left((N C)_{S 1}^{E}, E_{1}, X_{1}\right) \cup_{R}\left(\left((N C)_{S 2}^{E}, E_{2}, X_{2}\right)\right) \cap_{R}\left((N C)_{S 2}^{E}, E_{2}, X_{2}\right)\right)=\left((N C)_{S 1}^{E}, E_{1}, X_{1}\right)$, (iv) $\left((N C)_{S 1}^{E}, E_{1}, X_{1}\right) \cap_{R}\left(\left((N C)_{S 2}^{E}, E_{2}, X_{2}\right) \cup_{R}\left((N C)_{S 2}^{E}, E_{2}, X_{2}\right)\right)=\left((N C)_{S 1}^{E}, E_{1}, X_{1}\right)$.

Proof. The proof is straightforward.

Next we define some more operations on NCSESs.

Definition 21. For two neutrosophic cubic soft expert sets (NCSESs) $\left((N C)_{S 1}^{E}, E_{1}, X_{1}\right)$ and $\left((N C)_{S 2}^{E}, E_{2}, X_{2}\right)$ over $U, P$-AND is denoted as

$$
\left((N C)_{S 1}^{E}, E_{1}, X_{1}\right) \bigwedge_{P}\left((N C)_{S 2}^{E}, E_{2}, X_{2}\right)=\left((N C)_{S 3}^{E},\left(E_{1} \times E_{2}\right),\left(X_{1} \times X_{2}\right)\right),
$$

where

$$
\begin{aligned}
& \left((N C)_{S 3}^{E},\left(E_{1} \times E_{2}\right),\left(X_{1} \times X_{2}\right)\right)=(N C)_{S 3}^{E}((e, f),(x, y)) \\
& =(N C)_{S 1}^{E}(e, x) \cap_{P}(N C)_{S 2}^{E}(f, y) \\
& \left.=\left\{\left\langle u, I_{1(e, x)}^{N}(u), N_{1(e, x)}(u)\right\rangle, u \in U\right\},(e, x) \in E_{1} \times X_{1}\right\} \\
& \text { and } \left.\left\{\left\langle u, I_{2(e, x)}^{N}(u), N_{2(e, x)}(u)\right\rangle, u \in U\right\},(e, x) \in E_{1} \times X_{1}\right\} \text {. }
\end{aligned}
$$

Definition 22. For any two NCSESS $\left((N C)_{S 1}^{E}, E_{1}, X_{1}\right)$ and $\left((N C)_{S 2}^{E}, E_{2}, X_{2}\right)$ over $U, R-A N D$ is denoted as

$$
\left((N C)_{S 1}^{E}, E_{1}, X_{1}\right) \bigwedge_{R}\left((N C)_{S 2}^{E}, E_{2}, X_{2}\right)=\left((N C)_{S 3}^{E},\left(E_{1} \times E_{2}\right),\left(X_{1} \times X_{2}\right)\right),
$$

where

$$
\begin{aligned}
& \left((N C)_{S 3}^{E},\left(E_{1} \times E_{2}\right),\left(X_{1} \times X_{2}\right)\right)=(N C)_{S 3}^{E}((e, f),(x, y))=(N C)_{S 1}^{E}(e, x) \cap_{R}(N C)_{S 2}^{E}(f, y) \\
& \left.=\left\{\left\langle u, I_{1(e, x)}^{N}(u), N_{1(e, x)}(u)\right\rangle, u \in U\right\},(e, x) \in E_{1} \times X_{1}\right\} \\
& \text { and } \left.\left\{\left\langle u, I_{2(e, x)}^{N}(u), N_{2(e, x)}(u)\right\rangle, u \in U\right\},(e, x) \in E_{1} \times X_{1}\right\} \text {. }
\end{aligned}
$$

Definition 23. For any two NCSESS $\left((N C)_{S 1}^{E}, E_{1}, X_{1}\right)$ and $\left((N C)_{S 2}^{E}, E_{2}, X_{2}\right)$ over $U, P-O R$ is denoted as

$$
\left((N C)_{S 1}^{E}, E_{1}, X_{1}\right) \bigvee_{P}\left((N C)_{S 2}^{E}, E_{2}, X_{2}\right)=\left((N C)_{S 3}^{E},\left(E_{1} \times E_{2}\right),\left(X_{1} \times X_{2}\right)\right)
$$


where

$$
\begin{aligned}
& \left((N C)_{S 3}^{E}\left(E_{1} \times E_{2}\right),\left(X_{1} \times X_{2}\right)\right)=(N C)_{S 3}^{E}((e, f),(x, y))=(N C)_{S 1}^{E}(e, x) \cap_{R}(N C)_{S 2}^{E}(f, y) \\
& \left.=\left\{\left\langle u, I_{1(e, x)}^{N}(u), N_{1(e, x)}(u)\right\rangle, u \in U\right\},(e, x) \in E_{1} \times X_{1}\right\} \\
& \text { and } \left.\left\{\left\langle u, I_{2(e, x)}^{N}(u), N_{2(e, x)}(u)\right\rangle, u \in U\right\},(e, x) \in E_{1} \times X_{1}\right\} \text {. }
\end{aligned}
$$

Definition 24. For any two NCSESs $\left((N C)_{S 1}^{E}, E_{1}, X_{1}\right)$ and $\left((N C)_{S 2}^{E}, E_{2}, X_{2}\right)$ over $U, R$-OR is denoted as

$$
\left((N C)_{S 1}^{E}, E_{1}, X_{1}\right) \bigvee_{R}\left((N C)_{S 2}^{E}, E_{2}, X_{2}\right)=\left((N C)_{S 3}^{E},\left(E_{1} \times E_{2}\right),\left(X_{1} \times X_{2}\right)\right)
$$

where

$$
\begin{aligned}
& \left((N C)_{S 3}^{E},\left(E_{1} \times E_{2}\right),\left(X_{1} \times X_{2}\right)\right)=(N C)_{S 3}^{E}((e, f),(x, y))=(N C)_{S 1}^{E}(e, x) \cap_{R}(N C)_{S 2}^{E}(f, y) \\
& \left.=\left\{\left\langle u, I_{1(e, x)}^{N}(u), N_{1(e, x)}(u)\right\rangle, u \in U\right\},(e, x) \in E_{1} \times X_{1}\right\} \\
& \text { and } \left.\left\{\left\langle u, I_{2(e, x)}^{N}(u), N_{2(e, x)}(u)\right\rangle, u \in U\right\},(e, x) \in E_{1} \times X_{1}\right\} \text {. }
\end{aligned}
$$

Theorem 11. Let $\left((N C)_{S 1}^{E}, E_{1}, X_{1}\right)$, be a neutrosophic cubic soft expert sets in $U$. If $\left((N C)_{S 1}^{E}, E_{1}, X_{1}\right)$, is an INCSESS (resp., ENCSESS). Then $\left((N C)_{S 1}^{E}, E_{1}, X_{1}\right)^{C}$ is INCSESs (resp., ENCSESs) respectively.

Proof. Since $\left((N C)_{S 1}^{E}, E_{1}, X_{1}\right)$, is an INCSESS (resp., ENCSESs) in $U$, so for any $e \in E_{1}$ we have

$$
\begin{aligned}
(N C)_{S 1}^{E}(e)= & \left\{\left\langle x, I_{(N C))_{S 1}^{E}\left(e_{i}\right)}^{N}(x), N_{(N C)_{S 1}^{E}\left(e_{i}\right)}(u)\right\rangle: u \in U\right\} \text {. As } \\
& I_{(N C))_{S 1}^{E}\left(e_{i}\right)}^{N-}(u) \leq N_{(N C)_{S 1}^{E}\left(e_{i}\right)}(u) \leq I_{(N C))_{S 1}^{E}\left(e_{i}\right)}^{N+}(u) \\
& \Rightarrow 1-I_{(N C)_{S 1}^{N+}\left(e_{i}\right)}(u) \leq 1-N_{(N C))_{S 1}^{E}\left(e_{i}\right)}(u) \leq 1-I_{(N C))_{S 1}^{E}\left(e_{i}\right)}^{N-}(u) .
\end{aligned}
$$

Hence $\left((N C)_{S 1}^{E}, E_{1}, X_{1}\right)^{C}$ is an INCSES. Also

$$
\begin{aligned}
N_{(N C)_{S 1}^{E}\left(e_{i}\right)}(u) & \notin\left(I_{(N C)_{S 1}^{E}\left(e_{i}\right)}^{N-}(u), I_{(N C)_{S 1}^{E}\left(e_{i}\right)}^{N+}(u)\right) \forall u \in U \\
& \Rightarrow\left(1-N_{(N C)_{S 1}^{E}\left(e_{i}\right)}(u) \notin\left(1-I_{(N C)_{S 1}^{E}\left(e_{i}\right)}^{N+}(u), 1-I_{(N C)_{S 1}^{E}\left(e_{i}\right)}^{N-}(u)\right)\right.
\end{aligned}
$$

Hence $\left((N C)_{S 1}^{E}, E_{1}, X_{1}\right)^{C}$ is an ENCSES.

\section{Applications}

In this section, we use NCSESs to construct an algorithm and applied it to a decision-making problem. The series between Pakistan and India remains a hot cake for cricket lovers and this cricket rivalry existed between them at the start of partition. The first series between the two teams took place in 1951-52, when Pakistan made a tour of India. India made a tour of Pakistan for the first time in 1954-55. Between 1962 and 1977, no cricket was played between the two countries owing to two major wars in 1965 and 1971. The 1999 Kargil War and the 2008 Mumbai terrorist attacks have also interrupted the game of cricket between the two nations. The growth of large expatriate populations from India and Pakistan across the world led to neutral venues such as the United Arab Emirates and Canada hosting several bilateral and multilateral ODI series involving the two teams. Tickets for The India-Pakistan match in the 2015 World Cup in Australia sold out in 12 min at the ticket counters.

We try to use NCSESs in India-Pakistan cricket rivalry to conclude which country is suffering more from the above mentioned conflicts. 
For this we first define the neutrosophic cubic soft expert weight average operator (NCSEWAO) and score function.

Definition 25. Let $\left((N C)_{S}^{E}, E, X\right)=\left\{(N C)_{S}^{E}(e, x)=\left\{\left\langle u, I_{(e, x)}^{N}(u), N_{(e, x)}(u)\right\rangle, u \in U\right\},(e, x) \in E \times X\right\}$ be a NCSESs. Then neutrosophic cubic soft expert weight average operator (NCSEWAO) is denoted and define as

$$
\begin{aligned}
& \mathbb{Q}_{w_{i}}\left(I_{(N C))_{S}^{E}\left(e_{i}\right)}^{N}(x), N_{(N C)_{S}^{E}\left(e_{i}\right)}(x)\right)
\end{aligned}
$$

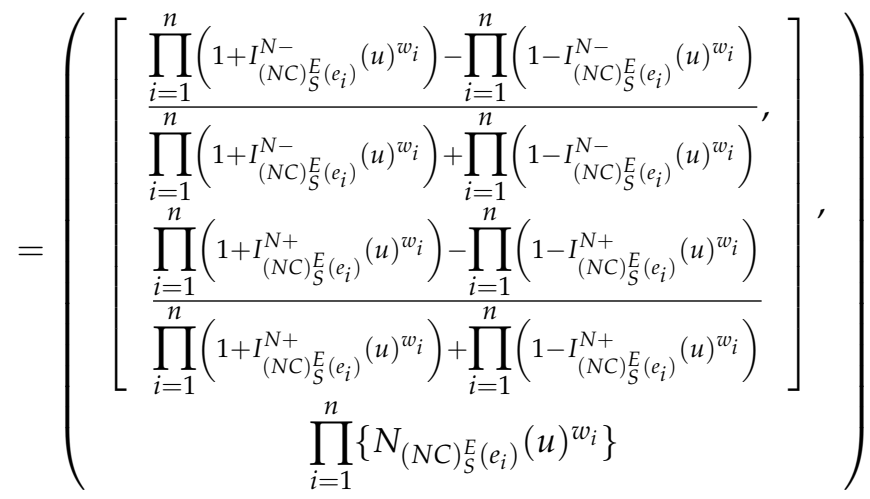

where $w_{i}$ is the weight of expert opinion, $w_{i} \in[0,1]$ and $\sum_{i=1}^{n} w_{i}=1$.

Definition 26. Let $(N C)_{S}^{E}=\left\langle\left[I_{(N C))_{S}^{E}\left(e_{i}\right)}^{N-}(u), I_{(N C))_{S}^{E}\left(e_{i}\right)}^{N+}(u)\right], N_{(N C))_{S}^{E}\left(e_{i}\right)}(u)\right\rangle$ be neutrosophic cubic soft expert value. A score function $Q$ of $(N C)_{S}^{E}$ is defined as $Q\left((N C)_{S}^{E}\right)=\frac{I_{(N C) S_{S}^{E}\left(e_{i}\right)}^{N-}(u)+I_{(N C) I_{S}^{N+}\left(e_{i}\right)}(u)-N_{(N C) E_{S}^{E}\left(e_{i}\right)}(u)}{9}$, where $Q\left((N C)_{S}^{E}\right) \in[-3,3]$.

Decision-making problems have been studied using fuzzy soft sets. Now, we are going to present multicriteria neutrosophic cubic soft set in decision-making along with weights and score function. For this we propose the following algorithmic steps as pictured in Figure 1.

Step 1: Define a decision problem by inputting the neutrosophic cubic soft expert set $(N C)_{S}^{E}\left(e_{i}, x_{i}\right)$.

Step 2: Use the opinions of experts in the form of NCSESS to determine the opinions regarding given criteria. Make a separate table for the opinion of each expert.

Step 3: Assign weight to each expert according to their expertise.

Step 4: Apply neutrosophic cubic soft expert weighted average operator to each above table and find the neutrosophic cubic soft expert weighted average corresponding to each attribute.

Step 5: Calculate the $\bigvee_{P}$ of $U_{j}$.

Step 6 : Calculate the score of each $U_{j}$.

Step 7: Generate the non-increasing order of all the alternatives according to their scores.

Example 10. Let $U=\left\{u_{1}=\right.$ India, $u_{2}=$ Pakistan $\}$ be the set of countries playing a cricket series, $E=\left\{e_{1}=\right.$ Pakistan Cricket Board (PCB), $e_{2}=$ The Board of Control for Cricket in India (BCCI), $e_{3}=$ Bilateral relations between Pakistan and India $\}$ be the set of factors affecting the series, $X=\{p, q, r\}$ be the set of experts giving their expert opinion. The expert may consider the most burning parameter as "ICC's Future Tours Programme $(F T P)^{\prime \prime}$ when they are giving their opinion by considering three times of frames as past, present, and future. 
Step 1: After a serious discussion, the committee constructed the following neutrosophic cubic soft expert set.

$$
\begin{aligned}
& (N C)_{S}^{E}\left(e_{1}, p\right)=\left\{\begin{array}{l}
\left(u_{1},[0.4,0.6],[0.2,0.5],[0.1,0.5], 0.3,0.1,0.7\right), \\
\left(u_{2},[0.6,0.7],[0.5,0.6],[0.6,0.8], 0.8,0.7,0.9\right),
\end{array}\right\} \\
& (N C)_{S}^{E}\left(e_{2}, p\right)=\left\{\begin{array}{l}
\left(u_{1},[0.5,0.7],[0.2,0.4],[0.1,0.4], 0.8,0.5,0.6\right), \\
\left(u_{2},[0.4,0.6],[0.3,0.5],[0.6,0.8], 0.7,0.2,0.4\right),
\end{array}\right\} \\
& (N C)_{S}^{E}\left(e_{3}, p\right)=\left\{\begin{array}{l}
\left(u_{1},[0.5,0.8],[0.1,0.3],[0.1,0.4], 0.4,0.4,0.5\right), \\
\left(u_{2},[0.1,0.3],[0.6,0.8],[0.4,0.6], 0.6,0.5,0.3\right),
\end{array}\right\} \\
& (N C)_{S}^{E}\left(e_{1}, q\right)=\left\{\begin{array}{l}
\left(u_{1},[0.5,0.8],[0.1,0.3],[0.1,0.4], 0.4,0.4,0.5\right), \\
\left(u_{2},[0.1,0.3],[0.6,0.8],[0.4,0.6], 0.6,0.5,0.3\right),
\end{array}\right\} \\
& (N C)_{S}^{E}\left(e_{2}, q\right)=\left\{\begin{array}{l}
\left(u_{1},[0.6,0.7],[0.3,0.4],[0.6,0.8], 0.8,0.7,0.5\right), \\
\left(u_{2},[0.3,0.5],[0.7,0.8],[0.6,0.7], 0.6,0.5,0.8\right),
\end{array}\right\} \\
& (N C)_{S}^{E}\left(e_{3}, q\right)=\left\{\begin{array}{l}
\left(u_{1},[0.4,0.6],[0.2,0.5],[0.1,0.5], 0.3,0.1,0.7\right), \\
\left(u_{2},[0.6,0.7],[0.5,0.6],[0.6,0.8], 0.8,0.7,0.9\right),
\end{array}\right\} \\
& (N C)_{S}^{E}\left(e_{1}, r\right)=\left\{\begin{array}{l}
\left(u_{1},[0.6,0.7],[0.3,0.4],[0.6,0.8], 0.8,0.7,0.5\right), \\
\left(u_{2},[0.3,0.5],[0.7,0.8],[0.6,0.7], 0.6,0.5,0.8\right),
\end{array}\right\} \\
& (N C)_{S}^{E}\left(e_{2}, r\right)=\left\{\begin{array}{l}
\left(u_{1},[0.4,0.6],[0.2,0.5],[0.1,0.5], 0.3,0.1,0.7\right), \\
\left(u_{2},[0.6,0.7],[0.5,0.6],[0.6,0.8], 0.8,0.7,0.9\right),
\end{array}\right\} \\
& (N C)_{S}^{E}\left(e_{3}, r\right)=\left\{\begin{array}{l}
\left(u_{1},[0.4,0.6],[0.2,0.5],[0.1,0.5], 0.3,0.1,0.7\right), \\
\left(u_{2},[0.6,0.7],[0.5,0.6],[0.6,0.8], 0.8,0.7,0.9\right),
\end{array}\right\}
\end{aligned}
$$

Step 2: Opinion of expert $p$

$$
\begin{aligned}
& \left(e_{1}, p\right)=\left\{\begin{array}{l}
\left(u_{1},[0.4,0.6],[0.2,0.5],[0.1,0.5], 0.3,0.1,0.7\right), \\
\left(u_{2},[0.6,0.7],[0.5,0.6],[0.6,0.8], 0.8,0.7,0.9\right),
\end{array}\right\} \\
& \left(e_{2}, p\right)=\left\{\begin{array}{l}
\left(u_{1},[0.5,0.7],[0.2,0.4],[0.1,0.4], 0.8,0.5,0.6\right), \\
\left(u_{2},[0.4,0.6],[0.3,0.5],[0.6,0.8], 0.7,0.2,0.4\right),
\end{array}\right\} \\
& \left(e_{3}, p\right)=\left\{\begin{array}{l}
\left(u_{1},[0.5,0.8],[0.1,0.3],[0.1,0.4], 0.4,0.4,0.5\right), \\
\left(u_{2},[0.1,0.3],[0.6,0.8],[0.4,0.6], 0.6,0.5,0.3\right),
\end{array}\right\}
\end{aligned}
$$

Opinion of expert $q$

$$
\begin{aligned}
& \left(e_{1}, q\right)=\left\{\begin{array}{l}
\left(u_{1},[0.5,0.8],[0.1,0.3],[0.1,0.4], 0.4,0.4,0.5\right), \\
\left(u_{2},[0.1,0.3],[0.6,0.8],[0.4,0.6], 0.6,0.5,0.3\right),
\end{array}\right\} \\
& \left(e_{2}, q\right)=\left\{\begin{array}{l}
\left(u_{1},[0.6,0.7],[0.3,0.4],[0.6,0.8], 0.8,0.7,0.5\right), \\
\left(u_{2},[0.3,0.5],[0.7,0.8],[0.6,0.7], 0.6,0.5,0.8\right),
\end{array}\right\} \\
& \left(e_{3}, q\right)=\left\{\begin{array}{l}
\left(u_{1},[0.4,0.6],[0.2,0.5],[0.1,0.5], 0.3,0.1,0.7\right), \\
\left(u_{2},[0.6,0.7],[0.5,0.6],[0.6,0.8], 0.8,0.7,0.9\right),
\end{array}\right\}
\end{aligned}
$$


Opinion of expert $r$

$$
\begin{aligned}
& \left(e_{1}, r\right)=\left\{\begin{array}{l}
\left(u_{1},[0.6,0.7],[0.3,0.4],[0.6,0.8], 0.8,0.7,0.5\right), \\
\left(u_{2},[0.3,0.5],[0.7,0.8],[0.6,0.7], 0.6,0.5,0.8\right),
\end{array}\right\} \\
& \left(e_{2}, r\right)=\left\{\begin{array}{l}
\left(u_{1},[0.4,0.6],[0.2,0.5],[0.1,0.5], 0.3,0.1,0.7\right), \\
\left(u_{2},[0.6,0.7],[0.5,0.6],[0.6,0.8], 0.8,0.7,0.9\right),
\end{array}\right\} \\
& \left(e_{3}, r\right)=\left\{\begin{array}{l}
\left(u_{1},[0.4,0.6],[0.2,0.5],[0.1,0.5], 0.3,0.1,0.7\right), \\
\left(u_{2},[0.6,0.7],[0.5,0.6],[0.6,0.8], 0.8,0.7,0.9\right),
\end{array}\right\}
\end{aligned}
$$

Step 3: Let $w=(0.5,0.25,0.25)$ be the weight vector assigned to the experts $p, q$ and $r$ respectively.

Step 4: The neutrosophic cubic soft expert weighted average (NCSEWA) of each attribute is displayed in Table 1.

Step 5: Calculate the $\bigvee_{P}$ of the first and second columns of Table 1 by using Definition 23. Thus we have

$$
\begin{aligned}
& U_{1}=\bigvee_{j=1}^{3}=([0.66,0.75],[0.80,0.99],[0.61,0.90],(0.95,0.92,0.91)) \\
& U_{2}=\bigvee_{j=1}^{3}=([0.97,0.99],[0.98,0.99],[0.98,0.99],(0.68,0.47,0.51))
\end{aligned}
$$

Step 6: Using Definition 26, we have $Q\left(u_{1}\right)=0.2244, Q\left(u_{2}\right)=0.4711$.

Step 7: The score of the NCSESS values corresponding to $\bigvee_{P}$ of $U_{j}$ implies the following order $u_{2}>u_{1}$.

Thus, we can conclude that the country $u_{2}=$ Pakistan is affected more by the factors, $e_{1}=$ PCB, $e_{2}=$ BCCI, $e_{3}=$ Bilateral relations between Pakistan and India.

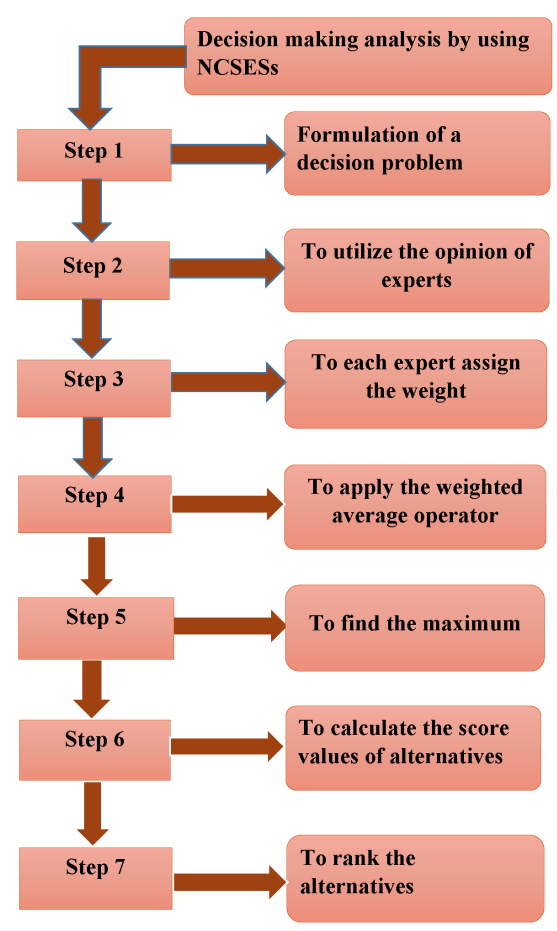

Figure 1. Flow chart of NCSESs based on MADM Problem. 
Table 1. NCSEWA of each attribute.

\begin{tabular}{ccc}
\hline & $u_{\mathbf{1}}$ & $\boldsymbol{u}_{\mathbf{2}}$ \\
\hline$e_{1} \quad\left(\begin{array}{c}([0.009,0.051],[0.77,0.87], \\
[0.61,0.90]), \\
(0.41,0.15,0.58)\end{array}\right)$ & $\left(\begin{array}{c}([0.97,0.98],[0.98,0.99], \\
[0.98,0.99]), \\
(0.52,0.14,0.19)\end{array}\right)$ \\
\hline & $e_{2} \quad\left(\begin{array}{c}([0.66,0.75],[0.40,0.49], \\
[0.54,0.80]), \\
(0.46,0.028,0.048)\end{array}\right)$ & $\left(\begin{array}{c}{[0.78,0.99],[0.57,0.86],} \\
{[0.80,0.99]} \\
(0.68,0.47,0.34)\end{array}\right)$, \\
$e_{3}$ & $\left(\begin{array}{c}([0.34,0.671],[0.80,0.99], \\
[0.23,0.71]), \\
(0.95,0.92,0.91)\end{array}\right)$
\end{tabular}

\section{Comparison Analysis}

In this paper, we extend the concept of soft expert sets to neutrosophic cubic soft expert sets. This new idea generalizes the different types of expert sets presented by Alkhazaleh and Salleh $[33,36]$, Broumi and Smarandache [40], Qayyum et al. [48] and Sahin et al. [43].

1. If we consider only truth part or indeterminacy part or falsity part, then our model and the model presented by Qayyum et al. [48] coincides with each other.

2. Since NCSESs consists of interval neutrosophic soft expert soft sets and neutrosophic soft expert sets and if we consider only the part containing the neutrosophic soft expert sets then our model reduces to the model presented by Sahin et al. [43].

3. If we consider the part containing the interval neutrosophic soft expert soft sets, we get a special class of soft expert sets.

4. Similarly imposing some extra conditions to our model it will reduce it to those models presented by Alkhazaleh and Salleh [33,36], Broumi and Smarandache [40].

\section{Conclusions}

The model of NCSESs can be used in many decision-making problems and it will produce more reliable results as compared to the previously defined versions of soft expert sets. This paper is of introductory nature where we establish this new generalized theory of soft expert sets with its basic properties and provide an application. In future we shall apply this model to other areas to illustrate its novelty. We have defined different operations of NCSESs including different versions of NCSESs. We have designed aggregation operators and score functions of neutrosophic cubic soft expert value. We have also constructed an algorithm based on this new concept and applied the developed approach to a numerical example.

The substantial impact of our outcome for the research field is that we gave a generalized form of soft expert system which certainly improves the decision-making theory due to its wide range of values in the form of truth, indeterminacy, and falsity. We have not used any software for the decision-making, since the preliminary research is on a small scale. We intend to expand the research to a larger scale on an applied problem so that we can develop a software and related interface. We also intend to apply the developed approach to a variety of games, signature theory and others, since the nature of NCSESs enables us to deal with vague and inconsistent data. In decision-making theory, we often deal with data which are inconsistent and vague. Thus, ex-ante decision-making problems can also be handled easily through NCSESs. In future, arguments and modern theories linked to behavioral patterns could strengthen the idea of NCSESs, especially using aggregation operators and methods that effectively deal with the uncertainty and inaccuracy of the input data [49].

Author Contributions: All authors contributed equally.

Funding: Universiti Kebangsaan Malaysia- Grant No. GUP-2017-105. 
Acknowledgments: We are indebted to Universiti Kebangsaan Malaysia for providing financial support under the grant GUP-2017-105.

Conflicts of Interest: The authors declare no conflict of interest.

\section{References}

1. Zadeh, L.A. Fuzzy sets. Inform. Control 1965, 8, 338-353. [CrossRef]

2. Turksen, I.B. Interval-valued fuzzy sets based on normal forms. Fuzzy Sets Syst. 1986, 20, 191-210. [CrossRef]

3. Atanassov, K. Intuitionistic fuzzy sets. Fuzzy Sets Syst. 1986, 20, 87-96. [CrossRef]

4. Atanassov, K.; Pasi, G.; Yager, R. Intuitionistic fuzzy interpretations of multi-criteria multi-person and multi-measurement tool decision making. Int. J. Syst. Sci. 2005, 36, 859-868. [CrossRef]

5. Smarandache, F. A Unifying Field in Logics: Neutrosophic Logic. Neutrosophy, Neutrosophic Set, Neutrosophic Probability; American Reserch Press: Rehoboth, IL, USA, 1999.

6. Smarandache, F. Neutrosophic set-a generalization of the intuitionistic fuzzy set. Int. J. Pure Appl. Math. 2005, 24, 287-297.

7. De, S.K.; Beg, I. Triangular dense fuzzy neutrosophic sets. Neutrosophic Sets Syst. 2016, 13, $26-37$.

8. Wang, H.; Smarandache, F.; Zhang, Y.Q.; Sunderraman, R. Interval Neutrosophic Sets and Logic: Theory and Applications in Computing; Hexis: Phoenix, AZ, USA, 2005.

9. Gulistan, M.; Nawaz, S.; Hassan, N. Neutrosophic triplet non-associative semihypergroups with application. Symmetry 2018, 10, 613. [CrossRef]

10. Jun, Y.B.; Kim, C.S.; Yang, K.O. Cubic Sets. Ann. Fuzzy Math. Inform. 2012, 4, 83-98.

11. Abughazalah, N.; Yaqoob, N. Applications of cubic structures to subsystems of finite state machines. Symmetry 2018, 10, 598. [CrossRef]

12. Rashid, S.; Yaqoob, N.; Akram, M.; Gulistan, M. Cubic graphs with application. Int. J. Anal. Appl. 2018, 16, 733-750.

13. Gulistan, M.; Yaqoob, N.; Vougiouklis, T.; Wahab, H.A. Extensions of cubic ideals in weak left almost semihypergroups. J. Intell. Fuzzy Syst. 2018, 34, 4161-4172. [CrossRef]

14. Ma, X.L.; Zhan, J.; Khan, M.; Gulistan, M.; Yaqoob, N. Generalized cubic relations in Hv-LA-semigroups. J. Discret. Math. Sci. Cryptogr. 2018, 21, 607-630. [CrossRef]

15. Yaqoob, N.; Gulistan, M.; Leoreanu-Fotea, V.; Hila, K. Cubic hyperideals in LA-semihypergroups. J. Intell. Fuzzy Syst. 2018, 34, 2707-2721. [CrossRef]

16. Gulistan, M.; Khan, M.; Yaqoob, N.; Shahzad, M. Structural properties of cubic sets in regular LA-semihypergroups. Fuzzy Inf. Eng. 2017, 9, 93-116. [CrossRef]

17. Khan, M.; Gulistan, M.; Yaqoob, N.; Hussain, F. General cubic hyperideals of LA-semihypergroups. Afr. Mat. 2016, 27, 731-751. [CrossRef]

18. Khan, M.; Jun, Y.B.; Gulistan, M.; Yaqoob, N. The generalized version of Jun's cubic sets in semigroups. J. Intell. Fuzzy Syst. 2015, 28, 947-960.

19. Yaqoob, N.; Mostafa, S.M.; Ansari, M.A. On cubic KU-ideals of KU-algebras. ISRN Algebra 2013, $2013,935905$. [CrossRef]

20. Aslam, M.; Aroob. T.; Yaqoob, N. On cubic $\Gamma$-hyperideals in left almost $\Gamma$-semihypergroups. Ann. Fuzzy Math. Inform. 2013, 5, 169-182.

21. Jun, Y.B. ; Smarandache, F.; Kim, C.S. Neutrosophic cubic sets. New Math. Nat. Comput. 2017, 13, 41-54. [CrossRef]

22. Khan, M.; Gulistan, M.; Yaqoob, N.; Shabir, M. Neutrosophic cubic $(\alpha, \beta)$-ideals in semigroups with application. J. Intell. Fuzzy Syst. 2018, 35, 2469-2483. [CrossRef]

23. Gulistan, M.; Yaqoob, N.; Rashid, Z.; Smarandache, F.; Wahab, H.A. A study on neutrosophic cubic graphs with real life applications in industries. Symmetry 2018, 10, 203. [CrossRef]

24. Gulistan, M.; Khan, A.; Abdullah, A.; Yaqoob, N. Complex neutrosophic subsemigroups and ideals. Int. J. Anal. Appl. 2018 16, 97-116.

25. Molodtsov, D.A. Soft set theory-first results. Comput. Math. Appl. 1999, 37, 19-31. [CrossRef]

26. Maji, P.K.; Roy, A.R. Soft set theory. Comput. Math. Appl. 2003, 45, 555-562. [CrossRef]

27. Maji, P.K.; Biswas, R.; Roy, A.R. Fuzzy soft sets. J. Fuzzy Math. 2001, 9, 589-602. 
28. Cagman, N.; Enginoglu, S.; Citak, F. Fuzzy soft set theory and its application. Iran. J. Fuzzy Syst. 2011, 8, 137-147.

29. Feng, F.; Jun, Y.B.; Liu, X.; Li. L. An adjustable approach to fuzzy soft set based decision making. J. Comput. Appl. Math. 2010, 234, 10-20. [CrossRef]

30. Gorzalczany, M.B. A method of inference in approximate reasoning based on interval valued fuzzy sets. Fuzzy Sets Syst. 1987, 21, 1-17. [CrossRef]

31. Kong, Z.; Gao, L.; Wang, L. Comment on "A fuzzy soft set theoretic approach to decision making problems". J. Comput. Appl. Math. 2009, 223, 540-542. [CrossRef]

32. Roy, A.R.; Maji, P.K. A fuzzy soft set theoretic approach to decision making problems. J. Comput. Appl. Math. 2007, 203, 412-418. [CrossRef]

33. Alkhazaleh, S.; Salleh, A.R. Soft expert sets. Adv. Decis. Sci. 2011, 2011, 757868. [CrossRef]

34. Arokia Lancy, A.; Tamilarasi, C.; Arockiarani, I. Fuzzy parameterization for decision making in risk management system via soft expert set. Int. J. Innov. Res. Stud. 2013, 2, 339-344.

35. Arokia Lancy, A.; Arockiarani, I. A fusion of soft expert set and matrix models. Int. J. Res. Eng. Technol. 2013, 2, 531-535.

36. Alkhazaleh, S.; Salleh, A.R. Fuzzy soft expert set and its application. Appl. Math. 2014, 5, 1349-1368. [CrossRef]

37. Bashir, M.; Salleh, A.R. Fuzzy parameterized soft expert set. Abstr. Appl. Anal. 2012, 2012, 25836. [CrossRef]

38. Bashir, M.; Salleh, A.R. Possibility fuzzy soft expert set. Open J. Appl. Sci. 2012, 12, 208-211. [CrossRef]

39. Alhazaymeh, K.; Hassan, N. Application of generalized vague soft expert set in decision making. Int. J. Pure Appl. Math. 2014, 93, 361-367. [CrossRef]

40. Broumi, S.; Smarandache, F. Intuitionistic fuzzy soft expert sets and its application in decision making. J. New Theory 2015, 1, 89-105.

41. Abu Qamar, M.; Hassan, N. Q-neutrosophic soft relation and its application in decision making. Entropy 2018, 20, 172. [CrossRef]

42. Abu Qamar, M.; Hassan, N. Entropy measures of distance and similarity of Q-neutrosophic soft sets and some applications. Entropy 2018, 20, 672. [CrossRef]

43. Sahin, M.; Alkhazaleh, S.; Ulucay, V. Neutrosophic soft expert sets. Appl. Math. 2015, 6, 116-127. [CrossRef]

44. Uluçay, V.; Sahin, M.; Hassan, N. Generalized neutrosophic soft expert set for multiple-criteria decision-making. Symmetry 2018, 10, 437. [CrossRef]

45. Al-Quran, A.; Hassan, N. Neutrosophic vague soft expert set theory. J. Intell. Fuzzy Syst. 2016, 30, 3691-3702. [CrossRef]

46. Al-Quran, A.; Hassan, N. The complex neutrosophic soft expert set and its application in decision making. J. Intell. Fuzzy Syst. 2018, 34, 569-582 [CrossRef]

47. Al-Quran, A.; Hassan, N. The complex neutrosophic soft expert relation and its multiple attribute decision-making method. Entropy 2018, 20, 101. [CrossRef]

48. Qayyum, A.; Abdullah, S.; Aslam, M. Cubic soft expert sets and their application in decision making. J. Intell. Fuzzy Syst. 2016, 31, 1585-1596. [CrossRef]

49. Ziemba, P.; Becker, J. Analysis of the digital divide using fuzzy forecasting. Symmetry 2019, 11, 166. [CrossRef]

(C) 2019 by the authors. Licensee MDPI, Basel, Switzerland. This article is an open access article distributed under the terms and conditions of the Creative Commons Attribution (CC BY) license (http://creativecommons.org/licenses/by/4.0/). 